\title{
Measurement of $V^{0}$ production ratios in $p p$ collisions at $\sqrt{s}=0.9$ and $7 \mathrm{TeV}$
}

\section{LHCb}

\section{The LHCb collaboration}

AbStract: The $\bar{\Lambda} / \Lambda$ and $\bar{\Lambda} / K_{\mathrm{S}}^{0}$ production ratios are measured by the LHCb detector from $0.3 \mathrm{nb}^{-1}$ of $p p$ collisions delivered by the LHC at $\sqrt{s}=0.9 \mathrm{TeV}$ and $1.8 \mathrm{nb}^{-1}$ at $\sqrt{s}=7 \mathrm{TeV}$. Both ratios are presented as a function of transverse momentum, $p_{\mathrm{T}}$, and rapidity, $y$, in the ranges $0.15<p_{\mathrm{T}}<2.50 \mathrm{GeV} / c$ and $2.0<y<4.5$. Results at the two energies are in good agreement as a function of rapidity loss, $\Delta y=y_{\text {beam }}-y$, and are consistent with previous measurements. The ratio $\bar{\Lambda} / \Lambda$, measuring the transport of baryon number from the collision into the detector, is smaller in data than predicted in simulation, particularly at high rapidity. The ratio $\bar{\Lambda} / K_{\mathrm{S}}^{0}$, measuring the baryon-to-meson suppression in strange quark hadronisation, is significantly larger than expected.

KEYwORDS: Hadron-Hadron Scattering

ARXIV EPRINT: 1107.0882 


\section{Contents}

1 Introduction $\quad 1$

2 The $\mathrm{LHCb}$ detector and data samples 2

3 Analysis procedure $\quad 3$

4 Systematic uncertainties $\quad 6$

$\begin{array}{llr}5 & \text { Results } & 10\end{array}$

6 Conclusions 12

$\begin{array}{ll}\text { A Tabulated results } & 13\end{array}$

$\begin{array}{ll}\text { B Tabulated results before non-prompt correction } & 15\end{array}$

$\begin{array}{ll}\text { The LHCb collaboration } & 17\end{array}$

\section{Introduction}

While the underlying interactions of hadronic collisions and hadronisation are understood within the Standard Model, exact computation of the processes governed by QCD are difficult due to the highly non-linear nature of the strong force. In the absence of full calculations, generators based on phenomenological models have been devised and optimised, or "tuned", to accurately reproduce experimental observations. These generators predict how Standard Model physics will behave at the LHC and constitute the reference for discoveries of New Physics effects.

Strange quark production is a powerful probe for hadronisation processes at $p p$ colliders since protons have no net strangeness. Recent experimental results in the field have been published by STAR [1] from RHIC $p p$ collisions at $\sqrt{s}=0.2 \mathrm{TeV}$ and by ALICE [2], CMS [3] and LHCb [4] from LHC $p p$ collisions at $\sqrt{s}=0.9$ and $7 \mathrm{TeV}$. LHCb can make an important contribution thanks to a full instrumentation of the detector in the forward region that is unique among the LHC experiments. Studies of data recorded at different energies with the same apparatus help to control the experimental systematic uncertainties.

In this paper we report on measurements of the efficiency corrected production ratios of the strange particles $\bar{\Lambda}, \Lambda$ and $K_{\mathrm{S}}^{0}$ as observables related to the fundamental processes behind parton fragmentation and hadronisation. The ratios

$$
\frac{\bar{\Lambda}}{\Lambda}=\frac{\sigma(p p \rightarrow \bar{\Lambda} X)}{\sigma(p p \rightarrow \Lambda X)}
$$


and

$$
\frac{\bar{\Lambda}}{K_{\mathrm{S}}^{0}}=\frac{\sigma(p p \rightarrow \bar{\Lambda} X)}{\sigma\left(p p \rightarrow K_{\mathrm{S}}^{0} X\right)}
$$

have predicted dependences on rapidity, $y$, and transverse momentum, $p_{\mathrm{T}}$, which can vary strongly between different tunes of the generators.

Measurements of the ratio $\bar{\Lambda} / \Lambda$ allow the study of the transport of baryon number from $p p$ collisions to final state hadrons and the ratio $\bar{\Lambda} / K_{\mathrm{S}}^{0}$ is a measure of baryon-to-meson suppression in strange quark hadronisation.

\section{The $\mathrm{LHCb}$ detector and data samples}

The Large Hadron Collider beauty experiment (LHCb) at CERN is a single-arm spectrometer covering the forward rapidity region. The analysis presented in this paper relies exclusively on the tracking detectors. The high precision tracking system begins with a silicon strip Vertex Locator (VELO), designed to identify displaced secondary vertices up to about $65 \mathrm{~cm}$ downstream of the nominal interaction point. A large area silicon tracker follows upstream of a dipole magnet and tracker stations, built with a mixture of straw tube and silicon strip detectors, are located downstream. The LHCb coordinate system is defined to be right-handed with its origin at the nominal interaction point, the $z$ axis aligned along the beam line towards the magnet and the $y$ axis pointing upwards. The bending plane is horizontal and the magnet has a reversible field, with the positive $B_{y}$ polarity called "up" and the negative "down". Tracks reconstructed through the full spectrometer experience an integrated magnetic field of around $4 \mathrm{Tm}$. The detector is described in full elsewhere [5].

A loose minimum bias trigger is used for this analysis, requiring at least one track segment in the downstream tracking stations. This trigger is more than $99 \%$ efficient for offline selected events that contain at least two tracks reconstructed through the full system.

Complementary data sets were recorded at two collision energies of $\sqrt{s}=0.9$ and $7 \mathrm{TeV}$, with both polarities of the dipole magnet. An integrated luminosity of $0.3 \mathrm{nb}^{-1}$ (corresponding to 12.5 million triggers) was taken at the lower energy, of which $48 \%$ had the up magnetic field configuration. At the higher energy, $67 \%$ of a total $1.8 \mathrm{nb}^{-1}(110.3$ million triggers) was taken with field up.

At injection energy $(\sqrt{s}=0.9 \mathrm{TeV})$, the proton beams are significantly broadened spatially compared to the accelerated beams at $\sqrt{s}=7 \mathrm{TeV}$. To protect the detector, the two halves of the VELO are retracted along the $x$ axis from their nominal position of inner radius of $8 \mathrm{~mm}$ to the beam, out to $18 \mathrm{~mm}$, which results in a reduction of the detector acceptance at small angles to the beam axis by approximately 0.5 units of rapidity.

The beams collide with a crossing angle in the horizontal plane tuned to compensate for LHCb's magnetic field. The angle required varies as a function of beam configuration and for the data taking period covered by this study was set to $2.1 \mathrm{mrad}$ at $\sqrt{s}=0.9 \mathrm{TeV}$ 
and $270 \mu$ rad at $7 \mathrm{TeV}$. Throughout this analysis $V^{0}$ momenta and any derived quantity such as rapidity are computed in the centre-of-mass frame of the colliding protons.

Samples of Monte Carlo (MC) simulated events have been produced in close approximation to the data-taking conditions described above for estimation of efficiencies and systematic uncertainties. A total of 73 million simulated minimum bias events were used for this analysis per magnet polarity at $\sqrt{s}=0.9 \mathrm{TeV}$ and $60(69)$ million events at $7 \mathrm{TeV}$ for field up (down). LHCb MC simulations are described in ref. [6], with $p p$ collisions generated by Pyтнia 6 [7]. Emerging particles decay via EvtGen [8], with final state radiation handled by Рнотоs [9]. The resulting particles are transported through $\mathrm{LHCb}$ by GEant 4 [10], which models hits on the sensitive elements of the detector as well as interactions between the particles and the detector material. Secondary particles produced in these material interactions decay via GEANT 4.

Additional samples of five million minimum bias events were generated for studies of systematic uncertainties using PyтніA 6 variants Perugia 0 (tuned on experimental results from SPS, LEP and Tevatron) and Perugia NOCR (an extreme model of baryon transport) [11]. Similarly sized samples of Pyтнia 8 [12] minimum bias diffractive events were also generated, including both hard and soft diffraction ${ }^{1}$ [13].

\section{Analysis procedure}

$V^{0}$ hadrons are named after the "V"-shaped track signature of their dominant decays: $\Lambda \rightarrow p \pi^{-}, \bar{\Lambda} \rightarrow \bar{p} \pi^{+}$and $K_{\mathrm{S}}^{0} \rightarrow \pi^{+} \pi^{-}$, which are reconstructed for this analysis. Only tracks with quality $\chi^{2} /$ ndf $<9$ are considered, with the $V^{0}$ required to decay within the VELO and the daughter tracks to be reconstructed through the full spectrometer. Any oppositelycharged pair is kept as a potential $V^{0}$ candidate if it forms a vertex with $\chi^{2}<9$ (with one degree of freedom for a $V^{0}$ vertex). $\bar{\Lambda}, \Lambda$ and $K_{\mathrm{S}}^{0}$ candidates are required to have invariant masses within $\pm 50 \mathrm{MeV} / c^{2}$ of the PDG values [14]. This mass window is large compared to the measured mass resolutions of about $2 \mathrm{MeV} / c^{2}$ for $\Lambda(\bar{\Lambda})$ and $5 \mathrm{MeV} / c^{2}$ for $K_{\mathrm{S}}^{0}$.

Combinatorial background is reduced with a Fisher discriminant based on the impact parameters (IP) of the daughter tracks $\left(d^{ \pm}\right)$and of the reconstructed $V^{0}$ mother, where the impact parameter is defined as the minimum distance of closest approach to the nearest reconstructed primary interaction vertex measured in $\mathrm{mm}$. The Fisher discriminant:

$$
\mathcal{F}_{\mathrm{IP}}=a \log _{10}\left(d_{\mathrm{IP}}^{+} / 1 \mathrm{~mm}\right)+b \log _{10}\left(d_{\mathrm{IP}}^{-} / 1 \mathrm{~mm}\right)+c \log _{10}\left(V_{\mathrm{IP}}^{0} / 1 \mathrm{~mm}\right)
$$

is optimised for signal significance $(S / \sqrt{S+B})$ on simulated events after the above quality criteria. The cut value, $\mathcal{F}_{\mathrm{IP}}>1$, and coefficients, $a=b=-c=1$, were found to be suitable for $\bar{\Lambda}, \Lambda$ and $K_{\mathrm{S}}^{0}$ at both collision energies (figure 1 ).

The $\Lambda(\bar{\Lambda})$ signal significance is improved by a $\pm 4.5 \mathrm{MeV} / c^{2}$ veto around the PDG $K_{\mathrm{S}}^{0}$ mass after re-calculation of each candidate's invariant mass with an alternative $\pi^{+} \pi^{-}$ daughter hypothesis. A similar veto to remove $\Lambda(\bar{\Lambda})$ with a $p \pi^{-}\left(\bar{p} \pi^{+}\right)$hypothesis from the $K_{\mathrm{S}}^{0}$ sample is not found to improve significance so is not applied.

\footnotetext{
${ }^{1}$ Single- and double-diffractive process types are considered: 92-94 in PYTHIA 6.421, with soft diffraction, and 103-105 in Pүтнia 8.130, with soft and hard diffraction.
} 


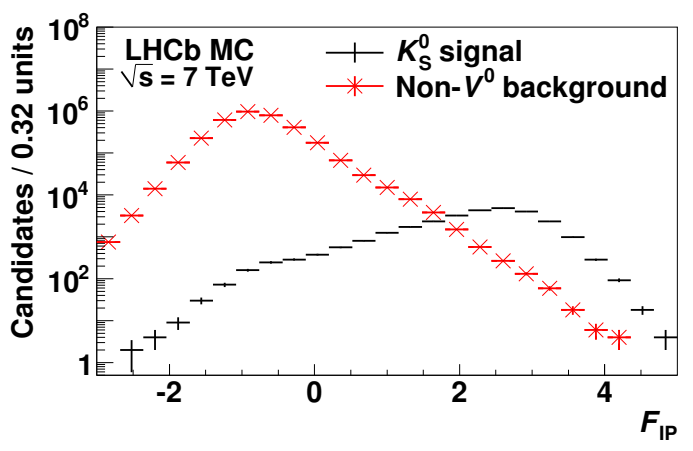

(a)

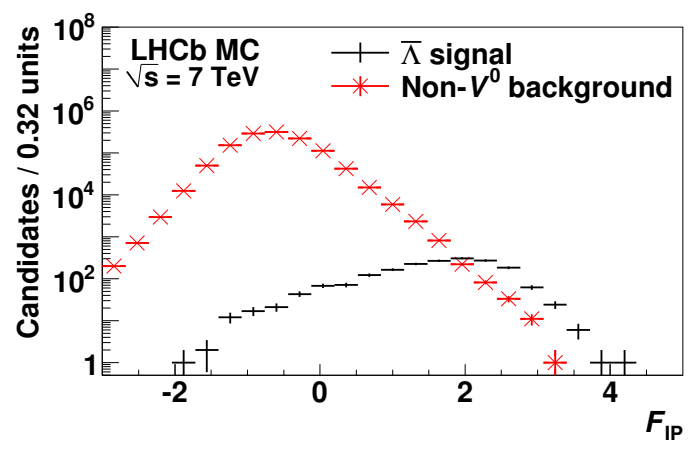

(b)

Figure 1. The Fisher discriminant $\mathcal{F}_{\mathrm{IP}}$ in 0.5 million Monte Carlo simulated minimum bias events at $\sqrt{s}=7 \mathrm{TeV}$ for (a) $K_{\mathrm{S}}^{0}$ and (b) $\bar{\Lambda}$.

\begin{tabular}{|c|cc|cc|}
\hline$\sqrt{s}$ & \multicolumn{2}{|c|}{$0.9 \mathrm{TeV}$} & \multicolumn{2}{c|}{$7 \mathrm{TeV}$} \\
Magnetic field & $\mathrm{Up}$ & Down & $\mathrm{Up}$ & Down \\
\hline $\bar{\Lambda}$ & $3,440 \pm 60$ & $4,100 \pm 70$ & $258,930 \pm 640$ & $132,550 \pm 460$ \\
$\Lambda$ & $4,880 \pm 80$ & $5,420 \pm 80$ & $294,010 \pm 680$ & $141,860 \pm 460$ \\
$K_{\mathrm{S}}^{0}$ & $35,790 \pm 200$ & $40,230 \pm 220$ & $2,737,090 \pm 1,940$ & $1,365,990 \pm 1,370$ \\
\hline
\end{tabular}

Table 1. Integrated signal yields extracted by fits to the invariant mass distributions of selected $V^{0}$ candidates from data taken with magnetic field up and down at $\sqrt{s}=0.9$ and $7 \mathrm{TeV}$.

After the above selection, $V^{0}$ yields are estimated from data and simulation by fits to the invariant mass distributions, examples of which are shown in figure 2. These fits are carried out with the method of unbinned extended maximum likelihood and are parametrised by a double Gaussian signal peak (with a common mean) over a linear background. The mean values show a small, but statistically significant, deviation from the known $K_{\mathrm{S}}^{0}$ and $\Lambda$ $(\bar{\Lambda})$ masses [14], reflecting the status of the momentum-scale calibration of the experiment. The width of the peak is computed as the quadratic average of the two Gaussian widths, weighted by their signal fractions. This width is found to be constant as a function of $p_{\mathrm{T}}$ and increases linearly toward higher $y$, e.g. by $1.4(0.8) \mathrm{MeV} / c^{2}$ per unit rapidity for $K_{\mathrm{S}}^{0}(\Lambda$ and $\bar{\Lambda}$ ) at $\sqrt{s}=7 \mathrm{TeV}$. The resulting signal yields are listed in table 3 .

Significant differences are observed between $V^{0}$ kinematic variables reconstructed in data and in the simulation used for efficiency determination. These differences can produce a bias for the measurement of $\bar{\Lambda} / K_{\mathrm{S}}^{0}$ given the different production kinematics of the baryon and meson. Simulated $V^{0}$ candidates are therefore weighted to match the two-dimensional $p_{\mathrm{T}}, y$ distributions observed in data. These distributions are shown projected along both axes in figure 3 . The $V^{0}$ signal yield $p_{\mathrm{T}}, y$ distributions are estimated from selected data and Monte Carlo candidates using sideband subtraction. Two-dimensional fits, linear in both $p_{\mathrm{T}}$ and $y$, are made to the ratios data/MC of these yields independently for $\bar{\Lambda}, \Lambda$ and $K_{\mathrm{S}}^{0}$, for each magnet polarity and collision energy. The resulting functions are used to weight generated and selected $V^{0}$ candidates in the Monte Carlo simulation. These weights vary across the measured $p_{\mathrm{T}}, y$ range between 0.4 and 2.1, with typical values between 0.8 and 1.2. 


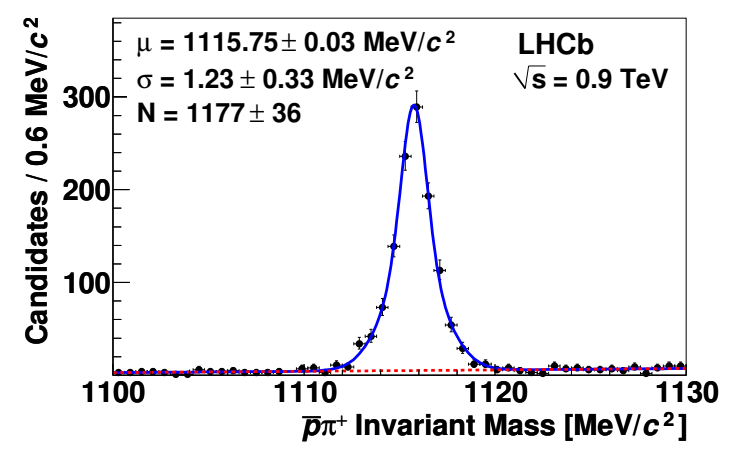

(a)

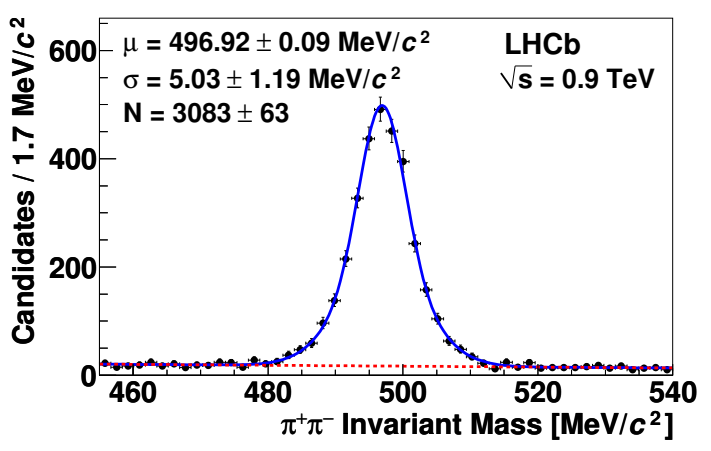

(b)

Figure 2. Invariant mass peaks for (a) $\bar{A}$ in the range $0.25<p_{\mathrm{T}}<2.50 \mathrm{GeV} / c \& 2.5<y<3.0$ and (b) $K_{\mathrm{S}}^{0}$ in the range $0.65<p_{\mathrm{T}}<1.00 \mathrm{GeV} / c \& 3.5<y<4.0$ at $\sqrt{s}=0.9 \mathrm{TeV}$ with field up. Signal yields, $N$, are found from fits (solid curves) with a double Gaussian peak with common mean, $\mu$, over a linear background (dashed lines). The width, $\sigma$, is computed as the quadratic average of the two Gaussian widths weighted by their signal fractions.

The measured ratios are presented in three complementary binning schemes: projections over the full $p_{\mathrm{T}}$ range, the full $y$ range, and a coarser two-dimensional binning. The rapidity range $2.0<y<4.0(4.5)$ is split into 0.5 -unit bins, while six bins in $p_{\mathrm{T}}$ are chosen to approximately equalise signal $V^{0}$ statistics in data over the range $0.25(0.15)<p_{\mathrm{T}}<2.50 \mathrm{GeV} / c$ from collisions at $\sqrt{s}=0.9(7) \mathrm{TeV}$. The two-dimensional binning combines pairs of $p_{\mathrm{T}}$ bins. The full analysis procedure is carried out independently in each $p_{\mathrm{T}}, y$ bin.

The efficiency for selecting prompt $V^{0}$ decays is estimated from simulation as

$$
\varepsilon=\frac{N\left(V^{0} \rightarrow d^{+} d^{-}\right)_{\text {Observed }}}{N\left(p p \rightarrow V^{0} X\right)_{\text {Generated }}}
$$

where the denominator is the number of prompt $V^{0}$ hadrons generated in a given $p_{\mathrm{T}}$, $y$ region after weighting and the numerator is the number of those weighted candidates found from the selection and fitting procedure described above. The efficiency therefore accounts for decays via other channels and losses from interactions with the detector material. Prompt $V^{0}$ hadrons are defined in Monte Carlo simulation by the cumulative lifetimes of their ancestors

$$
\sum_{i=1}^{n} c \tau_{i}<10^{-9} \mathrm{~m},
$$

where $\tau_{i}$ is the proper decay time of the $i^{\text {th }}$ ancestor. This veto is defined such as to keep only $V^{0}$ hadrons created either directly from the $p p$ collisions or from the strong or electromagnetic decays of particles produced at those collisions, removing $V^{0}$ hadrons generated from material interactions and weak decays. The Fisher discriminant $\mathcal{F}_{\text {IP }}$ strongly favours prompt $V^{0}$ hadrons, however a small non-prompt contamination in data would lead to a systematic bias in the ratios. The fractional contamination of selected events is determined from simulation to be $2-6 \%$ for $\bar{\Lambda}$ and $\Lambda$, depending on the measurement bin, and about 


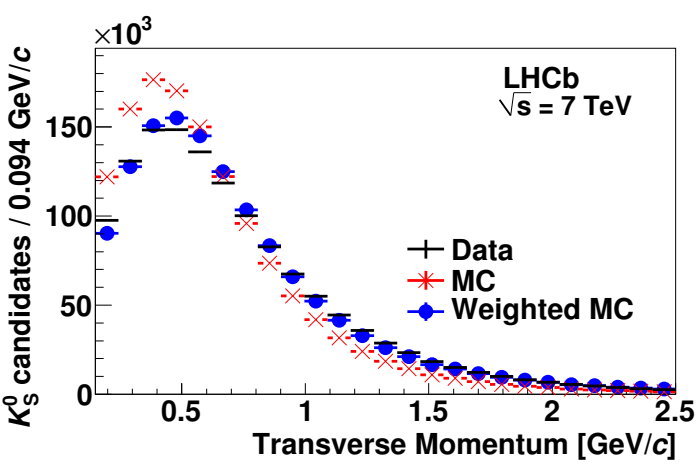

(a)

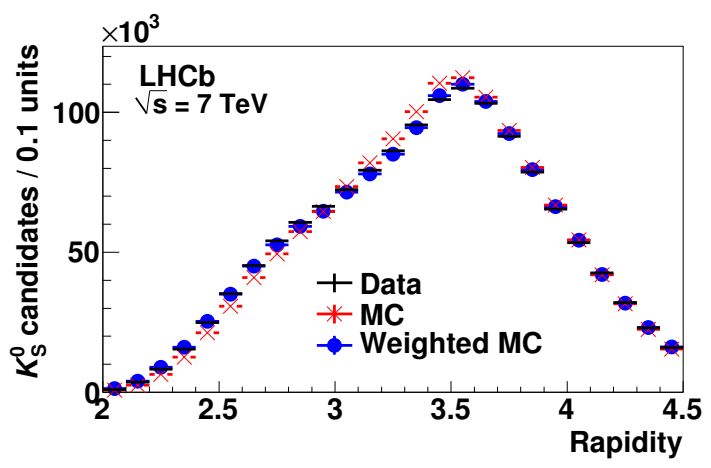

(b)

Figure 3. (a) Transverse momentum and (b) rapidity distributions for $K_{\mathrm{S}}^{0}$ in data and Monte Carlo simulation at $\sqrt{s}=7 \mathrm{TeV}$. The difference between data and Monte Carlo is reduced by weighting the simulated candidates.

$1 \%$ for $K_{\mathrm{S}}^{0}$. This effect is dominated by weak decays rather than material interactions. The resulting absolute corrections to the ratios $\bar{\Lambda} / \Lambda$ and $\bar{\Lambda} / K_{\mathrm{S}}^{0}$ are approximately 0.01 .

\section{Systematic uncertainties}

The measured efficiency corrected ratios $\bar{\Lambda} / \Lambda$ and $\bar{\Lambda} / K_{\mathrm{S}}^{0}$ are subsequently corrected for non-prompt contamination as found from Monte Carlo simulation and defined by eq. 3.3. This procedure relies on simulation and the corrections may be biased by the choice of the LHCb MC generator tune. To estimate a systematic uncertainty on the correction for non-prompt $V^{0}$, the contaminant fractions are also calculated using two alternative tunes of Pythia 6: Perugia 0 and Perugia NOCR [11]. The maximum differences in non-prompt fraction across the measurement range and at both energies are $<1 \%$ for each $V^{0}$ species. The resulting absolute uncertainties on the ratios are $<0.01$.

The efficiency of primary vertex reconstruction may introduce a bias on the measured ratios if the detector occupancy is different for events containing $K_{\mathrm{S}}^{0}, \Lambda$ or $\bar{\Lambda}$. This efficiency is compared in data and simulation using $V^{0}$ samples obtained with an alternative selection not requiring a primary vertex. Instead, the $V^{0}$ flight vector is extrapolated towards the beam axis to find the point of closest approach. The $z$ coordinate of this point is used to define a pseudo-vertex, with $x=y=0$. Candidates are kept if the impact parameters of their daughter tracks to this pseudo-vertex are $>0.2 \mathrm{~mm}$. There is a large overlap of signal candidates with the standard selection. The primary vertex finding efficiency is then explored by taking the ratio of these selected events which do or do not have a standard primary vertex. Calculated in bins of $p_{\mathrm{T}}$ and $y$, this efficiency agrees between data and simulation to better than $2 \%$ at both $\sqrt{s}=0.9$ and $7 \mathrm{TeV}$. The resulting absolute uncertainties on $\bar{\Lambda} / \Lambda$ and $\bar{\Lambda} / K_{\mathrm{S}}^{0}$ are $<0.02$ and $<0.01$, respectively.

The primary vertex finding algorithm requires at least three reconstructed tracks. ${ }^{2}$

\footnotetext{
${ }^{2}$ The minimum requirements for primary vertex reconstruction at $\mathrm{LHCb}$ can be approximated in Monte Carlo simulation by a generator-level cut requiring at least three charged particles from the collision with lifetime $c \tau>10^{-9} \mathrm{~m}$, momentum $p>0.3 \mathrm{GeV} / c$ and polar angle $15<\theta<460 \mathrm{mrad}$.
} 


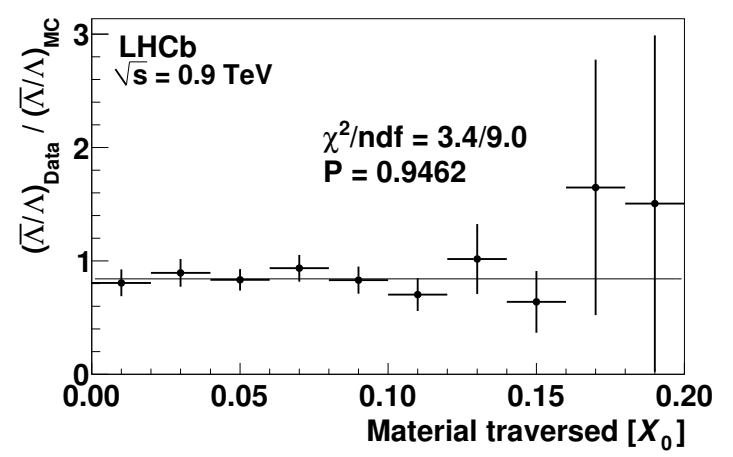

(a)

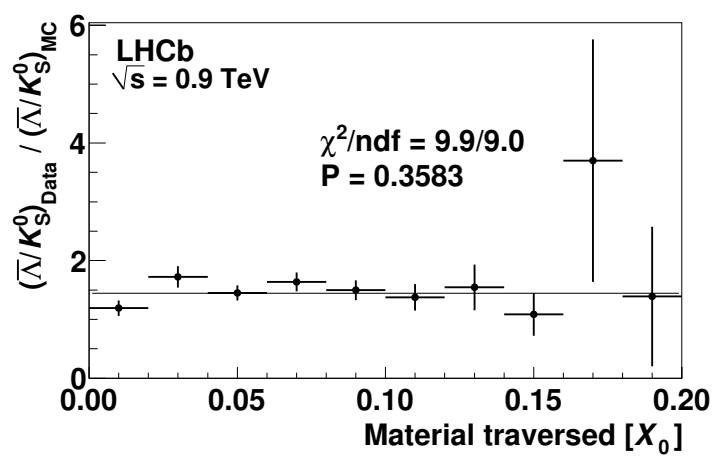

(b)

Figure 4. The double ratios $(\mathrm{a})(\bar{\Lambda} / \Lambda)_{\text {Data }} /(\bar{\Lambda} / \Lambda)_{\mathrm{MC}}$ and $(\mathrm{b})\left(\bar{\Lambda} / K_{\mathrm{S}}^{0}\right)_{\text {Data }} /\left(\bar{\Lambda} / K_{\mathrm{S}}^{0}\right)_{\mathrm{MC}}$ are shown as a function of the material traversed, in units of radiation length. Flat line fits, shown together with their respective $\chi^{2}$ probabilities, give no evidence of a bias.

Therefore, the reconstruction highly favours non-diffractive events due to the relatively low efficiency for finding diffractive interaction vertices, which tend to produce fewer tracks. In the LHCb MC simulation, the diffractive cross-section accounts for 28 (25) \% of the total minimum-bias cross-section of $65(91) \mathrm{mb}$ at $0.9(7) \mathrm{TeV}[6]$. Due to the primary vertex requirement, only about $3 \%$ of the $V^{0}$ candidates selected in simulation are produced in diffractive events. These fractions are determined using PутніA 6 which models only soft diffraction. As a cross check, the fractions are also calculated with PүтніA 8 which includes both soft and hard diffraction. The variation on the overall efficiency between models is about $2 \%$ for both ratios at $\sqrt{s}=7 \mathrm{TeV}$ and close to $1 \%$ at $0.9 \mathrm{TeV}$. Indeed, complete removal of diffractive events only produces a change of $0.01-0.02$ in the ratios across the measurement range.

The track reconstruction efficiency depends on particle momentum. In particular, the tracking efficiency varies rapidly with momentum for tracks below $5 \mathrm{GeV} / c$. Any bias is expected to be negligible for the ratio $\bar{\Lambda} / \Lambda$ but can be larger for $\bar{\Lambda} / K_{\mathrm{S}}^{0}$ due to the different kinematics. Two complementary procedures are employed to check this efficiency. First, track segments are reconstructed in the tracking stations upstream of the magnet. These track segments are then paired with the standard tracks reconstructed through the full detector and the pairs are required to form a $K_{\mathrm{S}}^{0}$ to ensure only genuine tracks are considered. This track matching gives a measure of the tracking efficiency for the upstream tracking systems. The second procedure uses the downstream stations to reconstruct track segments, which are similarly paired with standard tracks to measure the efficiency of the downstream tracking stations. The agreement between these efficiencies in data and simulation is better than $5 \%$. To estimate the resulting uncertainty on $\bar{\Lambda} / \Lambda$ and $\bar{\Lambda} / K_{\mathrm{S}}^{0}$, both ratios are re-calculated after weighting $V^{0}$ candidates by $95 \%$ for each daughter track with momentum below $5 \mathrm{GeV} / c$. The resulting systematic shifts in the ratios are $<0.01$.

Particle interactions within the detector are simulated using the GEANT 4 package, which implements interaction cross-sections for each particle according to the LHEP physics list [10]. These simulated cross-sections have been tested in the LHCb framework and are 


\begin{tabular}{|lcc|}
\hline Sources of systematic uncertainty & $\bar{\Lambda} / \Lambda$ & $\bar{\Lambda} / K_{\mathrm{S}}^{0}$ \\
\hline Correlated between field up and down : & 0.02 & 0.02 \\
Material interactions & $0.01-0.02$ & $0.01-0.02$ \\
Diffractive event fraction & $<0.02$ & $<0.01$ \\
Primary vertex finding & $<0.01$ & $<0.01$ \\
Non-prompt fraction & negligible & 0.01 \\
Track finding & \multicolumn{3}{|}{} \\
\hline Uncorrelated: & $0.01-0.05$ & $<0.03$ \\
Kinematic correction & 0.001 & 0.001 \\
Signal extraction from fit & $0.02-0.06$ & $0.02-0.03$ \\
\hline Total & & \\
\hline
\end{tabular}

Table 2. Absolute systematic errors are listed in descending order of importance. Ranges indicate uncertainties that vary across the measurement bins and/or by collision energy. Correlated sources of uncertainty between field up and down are identified.

consistent with the LHEP values. The small measured differences are propagated to $\bar{\Lambda} / \Lambda$ and $\bar{\Lambda} / K_{\mathrm{S}}^{0}$ to estimate absolute uncertainties on the ratios of about $0.02 . V^{0}$ absorption is limited by the requirement that each $V^{0}$ decay occurs within the most upstream tracker (the VELO). Secondary $V^{0}$ production in material is suppressed by the Fisher discriminant, which rejects $V^{0}$ candidates with large impact parameter. The potential bias on the ratios is explored by measurement of both $\bar{\Lambda} / \Lambda$ and $\bar{\Lambda} / K_{\mathrm{S}}^{0}$ as a function of material traversed (determined by the detector simulation), in units of radiation length, $X_{0}$. Data and simulation are compared by their ratio, shown in figure 4 . These double ratios are consistent with a flat line as a function of $X_{0}$, therefore any possible imperfections in the description of the detector material in simulation do not have a large effect on the $V^{0}$ ratios. Note that the double ratios are not expected to be unity since simulations do not predict the same values for $\bar{\Lambda} / \Lambda$ and $\bar{\Lambda} / K_{\mathrm{S}}^{0}$ as are observed in data.

The potential bias from the Fisher discriminant, $\mathcal{F}_{\mathrm{IP}}$, is investigated using a preselected sample, with only the track and vertex quality cuts applied. The distributions of $\mathcal{F}_{\mathrm{IP}}$ for $\Lambda, \bar{\Lambda}$ and $K_{\mathrm{S}}^{0}$ in data and Monte Carlo simulation are estimated using sideband subtraction. The double ratios of data/MC efficiencies are seen to be independent of the discriminant, implying that the distribution is well modelled in the simulation. No systematic uncertainty is assigned to this selection requirement.

A degradation is observed of the reconstructed impact parameter resolution in data compared to simulation. The simulated $V^{0}$ impact parameters are recalculated with smeared primary and secondary vertex positions to match the resolution measured in data. There is a negligible effect on the $V^{0}$ ratio results.

A good estimate of the reconstructed yields and their uncertainties in both data and simulation is provided by the fitting procedure but there may be a residual systematic uncertainty from the choice of this method. Comparisons are made using side-band subtraction and the resulting $V^{0}$ yields are in agreement with the results of the fits at the $0.1 \%$ level. The resulting absolute uncertainties on the ratios are on the order of 0.001 .

Simulated events are weighted to improve agreement between simulated $V^{0}$ kinematic 


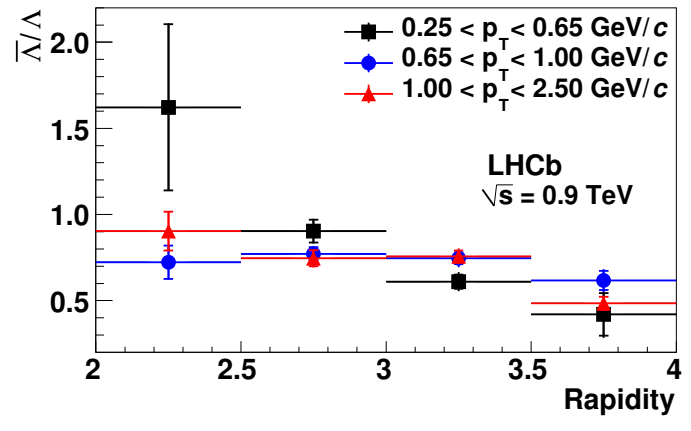

(a)

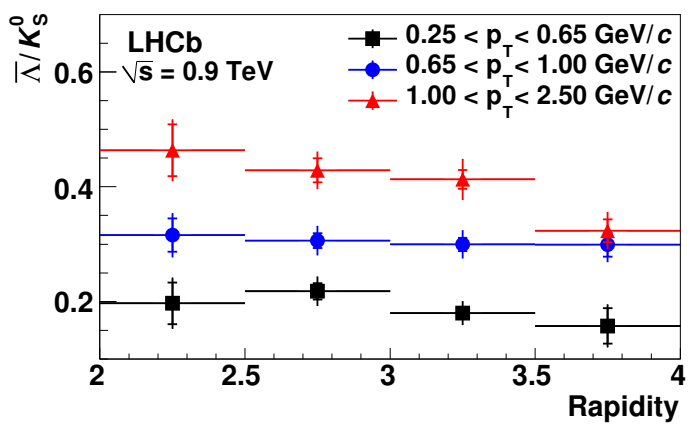

(c)

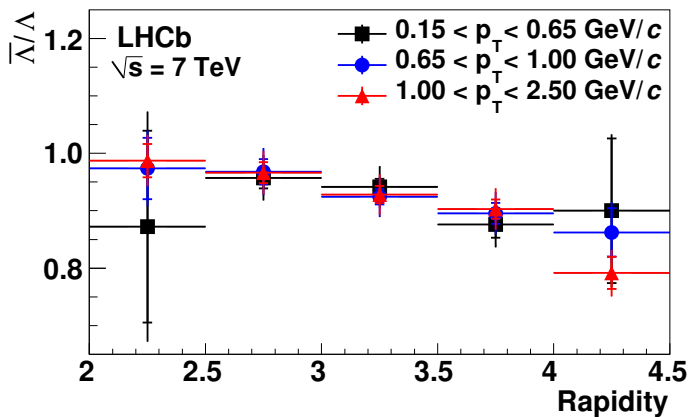

(b)

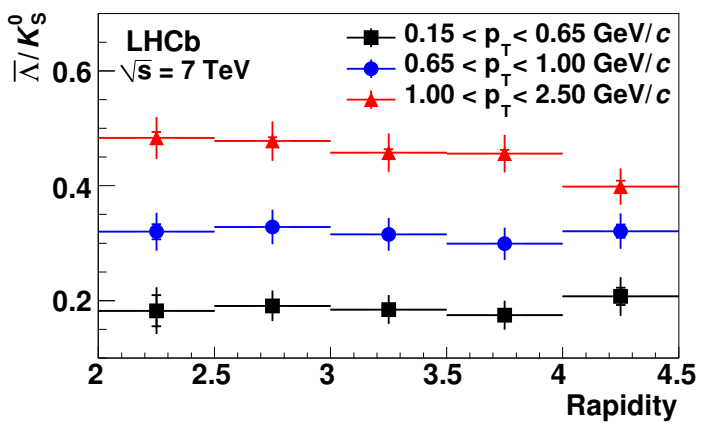

(d)

Figure 5. The ratios $\bar{\Lambda} / \Lambda$ and $\bar{\Lambda} / K_{\mathrm{s}}^{0}$ from the full analysis procedure at (a) \& (c) $\sqrt{s}=0.9 \mathrm{TeV}$ and (b) \& (d) $7 \mathrm{TeV}$ are shown as a function of rapidity, compared across intervals of transverse momentum. Vertical lines show the combined statistical and systematic uncertainties and the short horizontal bars (where visible) show the statistical component.

distributions and data. As described in section 3, these weights are calculated from a two-dimensional fit, linear in both $p_{\mathrm{T}}$ and $y$, to the distribution of the ratio between reconstructed data and simulated Monte Carlo candidates. This choice of parametrisation could be a source of systematic uncertainty, therefore alternative procedures are investigated including a two-dimensional polynomial fit to $3^{\text {rd }}$ order in both $p_{\mathrm{T}}$ and $y$ and a (non-parametric) bilinear interpolation. The results from each method are compared across the measurement range to estimate typical systematic uncertainties of $0.01-0.05$ for $\bar{\Lambda} / \Lambda$ and $<0.03$ for $\bar{\Lambda} / K_{\mathrm{S}}^{0}$.

The lifetime distributions of reconstructed and selected $V^{0}$ candidates are consistent between data and simulation. The possible influence of transverse $\Lambda(\bar{\Lambda})$ polarisation was explored by simulations with extreme values of polarisation and found to produce no significant effect on the measured ratios. Potential acceptance effects were checked as a function of azimuthal angle, with no evidence of systematic bias. The potential sources of systematic uncertainty or bias are summarised in table 4 . 


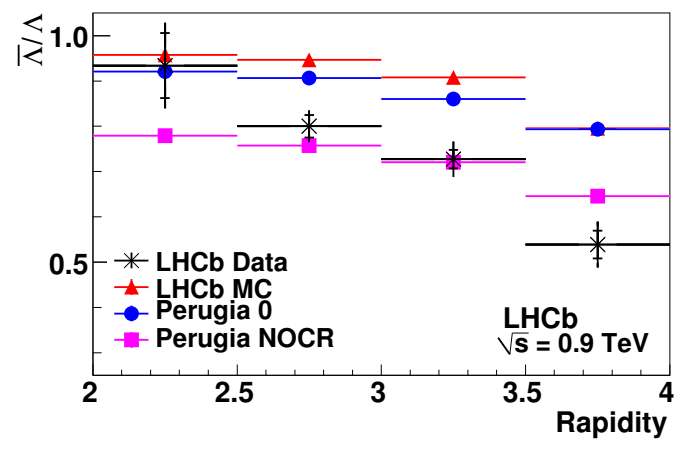

(a)

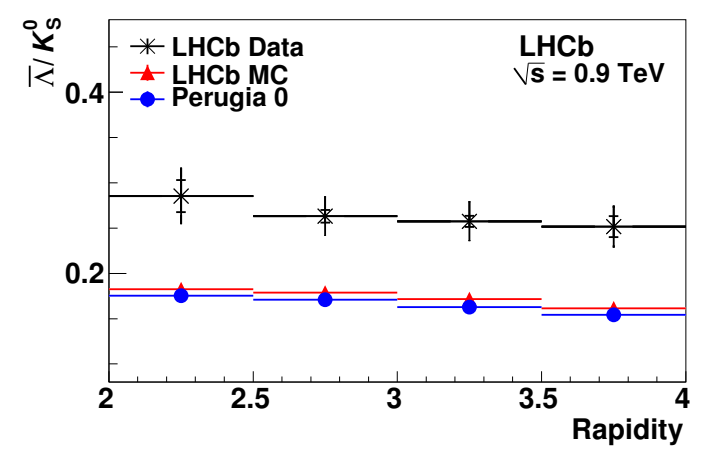

(c)

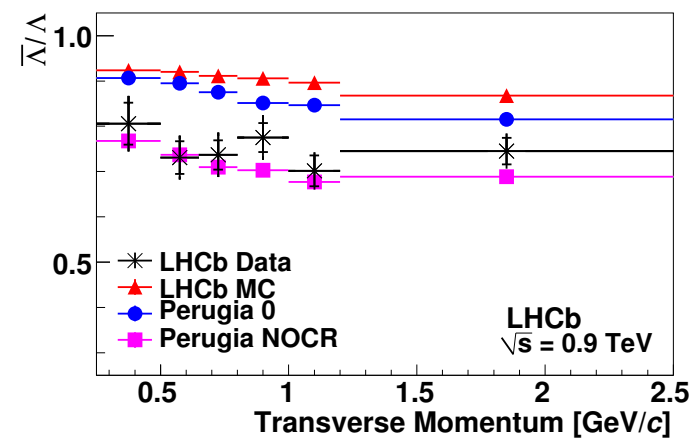

(b)

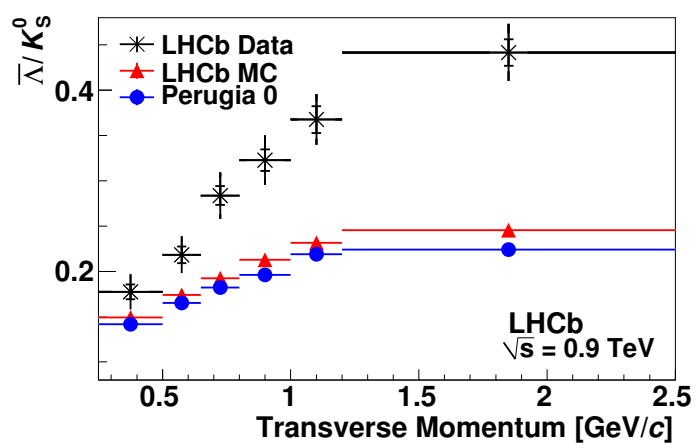

(d)

Figure 6. The ratios $\bar{\Lambda} / \Lambda$ and $\bar{\Lambda} / K_{\mathrm{S}}^{0}$ at $\sqrt{s}=0.9 \mathrm{TeV}$ are compared with the predictions of the LHCb MC, Perugia 0 and Perugia NOCR as a function of (a) \& (c) rapidity and (b) \& (d) transverse momentum. Vertical lines show the combined statistical and systematic uncertainties and the short horizontal bars (where visible) show the statistical component.

\section{Results}

The $\bar{\Lambda} / \Lambda$ and $\bar{\Lambda} / K_{\mathrm{S}}^{0}$ production ratios are measured independently for each magnetic field polarity. These measurements show good consistency after correction for detector acceptance. Bin-by-bin comparisons in the two-dimensional binning scheme give $\chi^{2}$ probabilities for $\bar{\Lambda} / \Lambda\left(\bar{\Lambda} / K_{\mathrm{S}}^{0}\right)$ of $3(18) \%$ at $\sqrt{s}=0.9 \mathrm{TeV}$ and $19(97) \%$ at $\sqrt{s}=7 \mathrm{TeV}$, with $12(15)$ degrees of freedom. The field up and down results are therefore combined to maximise statistical significance. A weighted average is computed such that the result has minimal variance while taking into account the correlations between sources of systematic uncertainty identified in table 4 . These combined results are shown as a function of $y$ in three intervals of $p_{\mathrm{T}}$ in figure 5 at $\sqrt{s}=0.9 \mathrm{TeV}$ and $7 \mathrm{TeV}$. The ratio $\bar{\Lambda} / K_{\mathrm{S}}^{0}$ shows a strong $p_{\mathrm{T}}$ dependence.

Both measured ratios are compared to the predictions of the PYTHIA 6 generator tunes: LHCb MC, Perugia 0 and Perugia NOCR, as functions of $p_{\mathrm{T}}$ and $y$ at $\sqrt{s}=0.9 \mathrm{TeV}$ (figure 6) and at $\sqrt{s}=7 \mathrm{TeV}$ (figure 7). According to Monte Carlo studies, as discussed in section 4 , the requirement for a reconstructed primary vertex results in only a small contribution from diffractive events to the selected $V^{0}$ sample, therefore non-diffractive simulated 


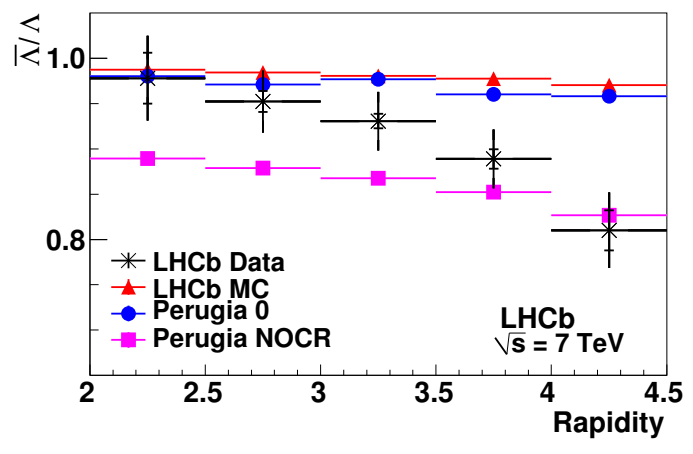

(a)

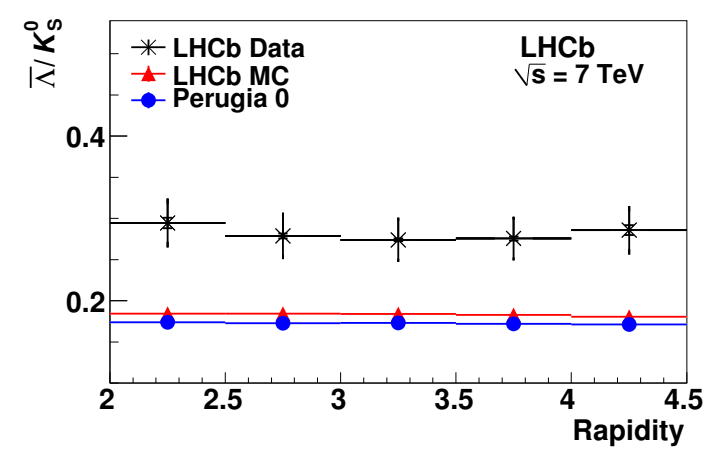

(c)

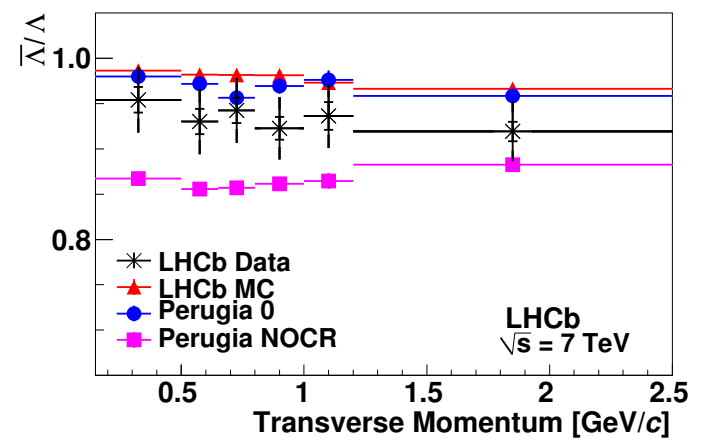

(b)

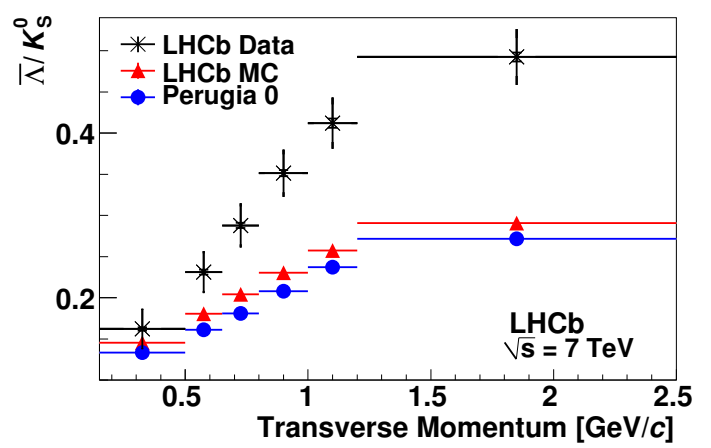

(d)

Figure 7. The ratios $\bar{\Lambda} / \Lambda$ and $\bar{\Lambda} / K_{\mathrm{S}}^{0}$ at $\sqrt{s}=7 \mathrm{TeV}$ compared with the predictions of the LHCb MC, Perugia 0 and Perugia NOCR as a function of (a) \& (c) rapidity and (b) \& (d) transverse momentum. Vertical lines show the combined statistical and systematic uncertainties and the short horizontal bars (where visible) show the statistical component.

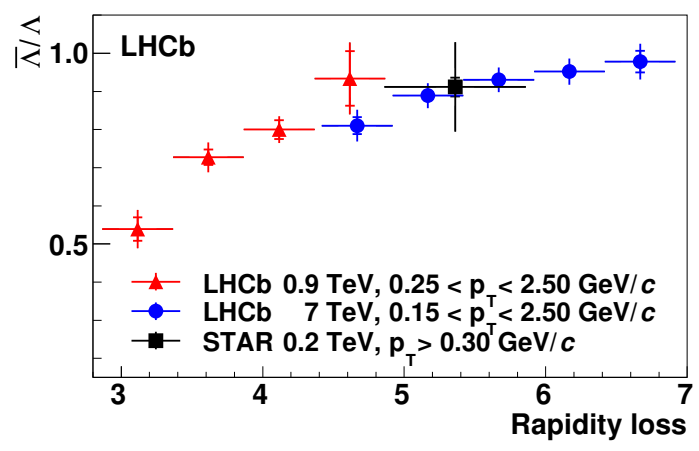

(a)

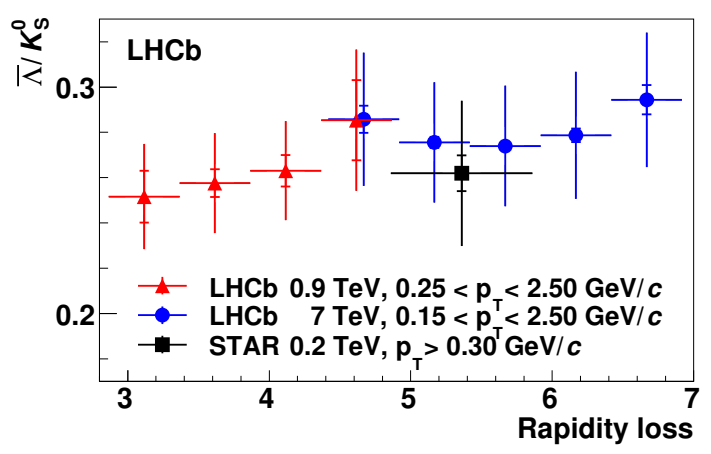

(b)

Figure 8. The ratios (a) $\bar{\Lambda} / \Lambda$ and (b) $\bar{\Lambda} / K_{\mathrm{S}}^{0}$ from LHCb are compared at both $\sqrt{s}=0.9 \mathrm{TeV}$ (triangles) and $7 \mathrm{TeV}$ (circles) with the published results from STAR [1] (squares) as a function of rapidity loss, $\Delta y=y_{\text {beam }}-y$. Vertical lines show the combined statistical and systematic uncertainties and the short horizontal bars (where visible) show the statistical component.

events are used for these comparisons. The predictions of LHCb MC and Perugia 0 are similar throughout. The ratio $\bar{\Lambda} / \Lambda$ is close to Perugia 0 at low $y$ but becomes smaller with 
higher rapidity, approaching Perugia NOCR. In collisions at $\sqrt{s}=7 \mathrm{TeV}$, this ratio is consistent with Perugia 0 across the measured $p_{\mathrm{T}}$ range but is closer to Perugia NOCR at $\sqrt{s}=0.9 \mathrm{TeV}$. The production ratio $\bar{\Lambda} / K_{\mathrm{S}}^{0}$ is larger in data than predicted by Perugia 0 at both collision energies and in all measurement bins, with the most significant differences observed at high $p_{\mathrm{T}}$.

To compare results at both collision energies, and to probe scaling violation, both production ratios are shown as a function of rapidity loss, $\Delta y=y_{\text {beam }}-y$, in figure 8 , where $y_{\text {beam }}$ is the rapidity of the protons in the anti-clockwise LHC beam, which travels along the positive $z$ direction through the detector. Excellent agreement is observed between results at both $\sqrt{s}=0.9$ and $7 \mathrm{TeV}$ as well as with results from STAR at $\sqrt{s}=0.2 \mathrm{TeV}$. The measured ratios are also consistent with results published by ALICE [2] and CMS [3].

The combined field up and down results are also given in tables in appendix A. Results without applying the model dependent non-prompt correction, as discussed in section 3, are shown for comparison in appendix B.

\section{Conclusions}

The ratio $\bar{\Lambda} / \Lambda$ is a measurement of the transport of baryon number from $p p$ collisions to final state hadrons. There is good agreement with Perugia 0 at low rapidity which is to be expected since the past experimental results used to test this model have focused on that rapidity region. At high rapidity however, the measurements favour the extreme baryon transport model of Perugia NOCR. The measured ratio $\bar{\Lambda} / K_{\mathrm{S}}^{0}$ is significantly larger than predicted by Perugia 0, i.e. relatively more baryons are produced in strange hadronisation in data than expected, particularly at higher $p_{\mathrm{T}}$. Similar results are found at both $\sqrt{s}=0.9$ and $7 \mathrm{TeV}$.

When plotted as a function of rapidity loss, $\Delta y$, there is excellent agreement between the measurements of both ratios at $\sqrt{s}=0.9$ and $7 \mathrm{TeV}$ as well as with STAR's results published at $0.2 \mathrm{TeV}$. The broad coverage of the measurements in $\Delta y$ provides a unique data set, which is complementary to previous results. The $V^{0}$ production ratios presented in this paper will help the development of hadronisation models to improve the predictions of Standard Model physics at the LHC which will define the baseline for new discoveries.

\section{Acknowledgments}

We express our gratitude to our colleagues in the CERN accelerator departments for the excellent performance of the LHC. We thank the technical and administrative staff at CERN and at the LHCb institutes, and acknowledge support from the National Agencies: CAPES, CNPq, FAPERJ and FINEP (Brazil); CERN; NSFC (China); CNRS/IN2P3 (France); BMBF, DFG, HGF and MPG (Germany); SFI (Ireland); INFN (Italy); FOM and NWO (Netherlands); SCSR (Poland); ANCS (Romania); MinES of Russia and Rosatom (Russia); MICINN, XUNGAL and GENCAT (Spain); SNSF and SER (Switzerland); NAS Ukraine (Ukraine); STFC (United Kingdom); NSF (USA). We also acknowledge the support received from the ERC under FP7 and the Région Auvergne. 


\section{A Tabulated results}

(a)

\begin{tabular}{|ccccc|}
\hline $\bar{\Lambda} / \Lambda$ & $2.0<y<2.5$ & $2.5<y<3.0$ & $3.0<y<3.5$ & $3.5<y<4.0$ \\
\hline $0.25<p_{\mathrm{T}}<2.50$ & $93.4 \pm 7.2 \pm 6.1$ & $80.0 \pm 2.5 \pm 2.5$ & $72.7 \pm 2.0 \pm 3.3$ & $53.9 \pm 3.1 \pm 4.0$ \\
\hline $0.25<p_{\mathrm{T}}<0.65$ & $162.2 \pm 48.2 \pm 6.6$ & $90.4 \pm 6.6 \pm 3.0$ & $61.0 \pm 4.2 \pm 3.5$ & $42.0 \pm 12.4 \pm 5.3$ \\
$0.65<p_{\mathrm{T}}<1.00$ & $72.3 \pm 9.7 \pm 2.5$ & $77.2 \pm 3.9 \pm 2.4$ & $74.6 \pm 3.3 \pm 3.9$ & $61.7 \pm 5.6 \pm 3.6$ \\
$1.00<p_{\mathrm{T}}<2.50$ & $90.4 \pm 11.3 \pm 2.8$ & $74.5 \pm 4.6 \pm 2.4$ & $75.7 \pm 3.4 \pm 3.1$ & $48.5 \pm 3.8 \pm 2.2$ \\
\hline
\end{tabular}

(b)

\begin{tabular}{|ccccc|}
\hline $\bar{\Lambda} / K_{\mathrm{S}}^{0}$ & $2.0<y<2.5$ & $2.5<y<3.0$ & $3.0<y<3.5$ & $3.5<y<4.0$ \\
\hline $0.25<p_{\mathrm{T}}<2.50$ & $28.5 \pm 1.8 \pm 2.6$ & $26.3 \pm 0.7 \pm 2.1$ & $25.8 \pm 0.6 \pm 2.1$ & $25.2 \pm 1.1 \pm 2.0$ \\
\hline $0.25<p_{\mathrm{T}}<0.65$ & $19.7 \pm 3.6 \pm 2.6$ & $21.8 \pm 1.4 \pm 2.2$ & $18.0 \pm 1.0 \pm 1.8$ & $15.8 \pm 3.1 \pm 2.1$ \\
$0.65<p_{\mathrm{T}}<1.00$ & $31.6 \pm 2.9 \pm 2.5$ & $30.6 \pm 1.3 \pm 2.3$ & $30.0 \pm 1.2 \pm 2.2$ & $29.9 \pm 2.1 \pm 2.2$ \\
$1.00<p_{\mathrm{T}}<2.50$ & $46.3 \pm 4.5 \pm 2.9$ & $42.9 \pm 2.1 \pm 2.5$ & $41.3 \pm 1.6 \pm 3.2$ & $32.3 \pm 2.0 \pm 2.6$ \\
\hline
\end{tabular}

(c)

\begin{tabular}{|ccc|}
\hline $2.0<y<4.0$ & $\bar{\Lambda} / \Lambda$ & $\bar{\Lambda} / K_{\mathrm{S}}^{0}$ \\
\hline $0.25<p_{\mathrm{T}}<0.50$ & $80.6 \pm 4.6 \pm 4.0$ & $17.7 \pm 0.8 \pm 1.7$ \\
$0.50<p_{\mathrm{T}}<0.65$ & $73.1 \pm 3.6 \pm 3.2$ & $21.8 \pm 0.9 \pm 1.8$ \\
$0.65<p_{\mathrm{T}}<0.80$ & $73.7 \pm 3.2 \pm 3.7$ & $28.4 \pm 1.0 \pm 2.3$ \\
$0.80<p_{\mathrm{T}}<1.00$ & $77.5 \pm 3.2 \pm 3.7$ & $32.3 \pm 1.2 \pm 2.4$ \\
$1.00<p_{\mathrm{T}}<1.20$ & $70.1 \pm 3.4 \pm 2.3$ & $36.8 \pm 1.5 \pm 2.4$ \\
$1.20<p_{\mathrm{T}}<2.50$ & $74.5 \pm 3.0 \pm 2.5$ & $44.2 \pm 1.5 \pm 2.8$ \\
\hline
\end{tabular}

Table 3. The production ratios $\bar{\Lambda} / \Lambda$ and $\bar{\Lambda} / K_{\mathrm{S}}^{0}$, measured at $\sqrt{s}=0.9 \mathrm{TeV}$, are quoted in percent with statistical and systematic errors as a function of (a) \& (b) rapidity, $y$, and (c) transverse momentum, $p_{\mathrm{T}}[\mathrm{GeV} / c]$. 
(a)

\begin{tabular}{|cccccc|}
\hline $\bar{\Lambda} / \Lambda$ & $2.0<y<2.5$ & $2.5<y<3.0$ & $3.0<y<3.5$ & $3.5<y<4.0$ & $4.0<y<4.5$ \\
\hline $0.15<p_{\mathrm{T}}<2.50$ & $97.8 \pm 2.8 \pm 3.8$ & $95.2 \pm 1.2 \pm 3.2$ & $93.1 \pm 0.8 \pm 3.1$ & $88.9 \pm 1.1 \pm 3.1$ & $81.0 \pm 2.2 \pm 3.5$ \\
\hline $0.15<p_{\mathrm{T}}<0.65$ & $87.2 \pm 16.7 \pm 11.0$ & $95.7 \pm 1.8 \pm 3.5$ & $94.2 \pm 1.4 \pm 3.3$ & $87.6 \pm 2.3 \pm 3.2$ & $90.0 \pm 12.6 \pm 4.2$ \\
$0.65<p_{\mathrm{T}}<1.00$ & $97.4 \pm 5.3 \pm 3.9$ & $96.8 \pm 2.2 \pm 3.5$ & $92.4 \pm 1.3 \pm 3.3$ & $89.6 \pm 1.8 \pm 3.2$ & $86.2 \pm 4.2 \pm 3.2$ \\
$1.00<p_{\mathrm{T}}<2.50$ & $98.7 \pm 2.9 \pm 3.4$ & $96.6 \pm 1.8 \pm 3.3$ & $92.8 \pm 1.5 \pm 3.2$ & $90.3 \pm 1.7 \pm 3.2$ & $79.2 \pm 2.8 \pm 2.9$ \\
\hline
\end{tabular}

(b)

\begin{tabular}{|cccccc|}
\hline $\bar{\Lambda} / K_{\mathrm{S}}^{0}$ & $2.0<y<2.5$ & $2.5<y<3.0$ & $3.0<y<3.5$ & $3.5<y<4.0$ & $4.0<y<4.5$ \\
\hline $0.15<p_{\mathrm{T}}<2.50$ & $29.4 \pm 0.6 \pm 2.9$ & $27.9 \pm 0.3 \pm 2.8$ & $27.4 \pm 0.2 \pm 2.7$ & $27.6 \pm 0.3 \pm 2.6$ & $28.6 \pm 0.6 \pm 2.9$ \\
\hline $0.15<p_{\mathrm{T}}<0.65$ & $18.2 \pm 2.7 \pm 3.0$ & $19.1 \pm 0.3 \pm 2.6$ & $18.5 \pm 0.2 \pm 2.5$ & $17.5 \pm 0.4 \pm 2.5$ & $20.7 \pm 1.5 \pm 3.0$ \\
$0.65<p_{\mathrm{T}}<1.00$ & $32.0 \pm 1.3 \pm 3.0$ & $32.8 \pm 0.6 \pm 3.0$ & $31.5 \pm 0.4 \pm 2.8$ & $29.9 \pm 0.5 \pm 2.8$ & $32.1 \pm 1.2 \pm 2.9$ \\
$1.00<p_{\mathrm{T}}<2.50$ & $48.3 \pm 1.1 \pm 3.5$ & $47.8 \pm 0.7 \pm 3.3$ & $45.8 \pm 0.6 \pm 3.3$ & $45.6 \pm 0.7 \pm 3.2$ & $39.9 \pm 1.0 \pm 3.0$ \\
\hline
\end{tabular}

(c)

\begin{tabular}{|ccc|}
\hline $2.0<y<4.5$ & $\bar{\Lambda} / \Lambda$ & $\bar{\Lambda} / K_{\mathrm{S}}^{0}$ \\
\hline $0.15<p_{\mathrm{T}}<0.50$ & $95.4 \pm 1.4 \pm 3.4$ & $16.2 \pm 0.2 \pm 2.4$ \\
$0.50<p_{\mathrm{T}}<0.65$ & $93.0 \pm 1.4 \pm 3.3$ & $23.1 \pm 0.3 \pm 2.5$ \\
$0.65<p_{\mathrm{T}}<0.80$ & $94.3 \pm 1.4 \pm 3.3$ & $28.8 \pm 0.3 \pm 2.7$ \\
$0.80<p_{\mathrm{T}}<1.00$ & $92.3 \pm 1.3 \pm 3.2$ & $35.1 \pm 0.4 \pm 2.8$ \\
$1.00<p_{\mathrm{T}}<1.20$ & $93.6 \pm 1.5 \pm 3.2$ & $41.2 \pm 0.6 \pm 3.0$ \\
$1.20<p_{\mathrm{T}}<2.50$ & $91.9 \pm 1.1 \pm 3.1$ & $49.2 \pm 0.5 \pm 3.4$ \\
\hline
\end{tabular}

Table 4. The production ratios $\bar{\Lambda} / \Lambda$ and $\bar{\Lambda} / K_{\mathrm{S}}^{0}$, measured at $\sqrt{s}=7 \mathrm{TeV}$, are quoted in percent with statistical and systematic errors as a function of (a) \& (b) rapidity, $y$, and (c) transverse momentum, $p_{\mathrm{T}}[\mathrm{GeV} / c]$. 


\section{B Tabulated results before non-prompt correction}

(a)

\begin{tabular}{|ccccc|}
\hline $\bar{\Lambda} / \Lambda$ & $2.0<y<2.5$ & $2.5<y<3.0$ & $3.0<y<3.5$ & $3.5<y<4.0$ \\
\hline $0.25<p_{\mathrm{T}}<2.50$ & $93.1 \pm 7.2 \pm 6.0$ & $79.3 \pm 2.5 \pm 2.4$ & $73.2 \pm 2.0 \pm 3.2$ & $54.1 \pm 3.1 \pm 3.9$ \\
\hline $0.25<p_{\mathrm{T}}<0.65$ & $163.7 \pm 48.2 \pm 6.5$ & $89.2 \pm 6.6 \pm 2.8$ & $61.5 \pm 4.2 \pm 3.4$ & $41.4 \pm 12.4 \pm 5.3$ \\
$0.65<p_{\mathrm{T}}<1.00$ & $71.8 \pm 9.7 \pm 2.4$ & $76.5 \pm 3.9 \pm 2.2$ & $75.2 \pm 3.3 \pm 3.8$ & $62.0 \pm 5.6 \pm 3.5$ \\
$1.00<p_{\mathrm{T}}<2.50$ & $89.9 \pm 11.3 \pm 2.7$ & $74.2 \pm 4.6 \pm 2.3$ & $75.7 \pm 3.4 \pm 3.0$ & $48.5 \pm 3.8 \pm 2.1$ \\
\hline
\end{tabular}

(b)

\begin{tabular}{|ccccc|}
\hline $\bar{\Lambda} / K_{\mathrm{S}}^{0}$ & $2.0<y<2.5$ & $2.5<y<3.0$ & $3.0<y<3.5$ & $3.5<y<4.0$ \\
\hline $0.25<p_{\mathrm{T}}<2.50$ & $28.9 \pm 1.8 \pm 2.4$ & $27.2 \pm 0.7 \pm 1.9$ & $26.6 \pm 0.6 \pm 1.9$ & $25.6 \pm 1.1 \pm 1.8$ \\
\hline $0.25<p_{\mathrm{T}}<0.65$ & $20.7 \pm 3.6 \pm 2.4$ & $23.0 \pm 1.4 \pm 2.0$ & $18.9 \pm 1.0 \pm 1.6$ & $16.3 \pm 3.1 \pm 1.9$ \\
$0.65<p_{\mathrm{T}}<1.00$ & $31.9 \pm 2.9 \pm 2.3$ & $31.5 \pm 1.3 \pm 2.1$ & $31.0 \pm 1.2 \pm 2.0$ & $30.6 \pm 2.1 \pm 2.0$ \\
$1.00<p_{\mathrm{T}}<2.50$ & $46.7 \pm 4.5 \pm 2.8$ & $43.1 \pm 2.1 \pm 2.4$ & $41.9 \pm 1.6 \pm 3.0$ & $32.5 \pm 2.0 \pm 2.4$ \\
\hline
\end{tabular}

(c)

\begin{tabular}{|ccc|}
\hline $2.0<y<4.0$ & $\bar{\Lambda} / \Lambda$ & $\bar{\Lambda} / K_{\mathrm{S}}^{0}$ \\
\hline $0.25<p_{\mathrm{T}}<0.50$ & $80.1 \pm 4.6 \pm 3.9$ & $18.8 \pm 0.8 \pm 1.5$ \\
$0.50<p_{\mathrm{T}}<0.65$ & $72.9 \pm 3.6 \pm 3.1$ & $22.9 \pm 0.9 \pm 1.6$ \\
$0.65<p_{\mathrm{T}}<0.80$ & $73.9 \pm 3.2 \pm 3.6$ & $29.5 \pm 1.0 \pm 2.1$ \\
$0.80<p_{\mathrm{T}}<1.00$ & $77.5 \pm 3.2 \pm 3.5$ & $33.1 \pm 1.2 \pm 2.3$ \\
$1.00<p_{\mathrm{T}}<1.20$ & $70.1 \pm 3.4 \pm 2.1$ & $37.2 \pm 1.5 \pm 2.2$ \\
$1.20<p_{\mathrm{T}}<2.50$ & $74.4 \pm 3.0 \pm 2.3$ & $44.5 \pm 1.5 \pm 2.6$ \\
\hline
\end{tabular}

Table 5. The production ratios $\bar{\Lambda} / \Lambda$ and $\bar{\Lambda} / K_{\mathrm{S}}^{0}$ without non-prompt corrections at $\sqrt{s}=0.9 \mathrm{TeV}$ are quoted in percent with statistical and systematic errors as a function of (a) \& (b) rapidity, $y$, and (c) transverse momentum, $p_{\mathrm{T}}[\mathrm{GeV} / \mathrm{c}]$. 
(a)

\begin{tabular}{|cccccc|}
\hline $\bar{\Lambda} / \Lambda$ & $2.0<y<2.5$ & $2.5<y<3.0$ & $3.0<y<3.5$ & $3.5<y<4.0$ & $4.0<y<4.5$ \\
\hline $0.15<p_{\mathrm{T}}<2.50$ & $97.3 \pm 2.8 \pm 3.6$ & $95.1 \pm 1.2 \pm 3.1$ & $92.7 \pm 0.8 \pm 3.0$ & $88.6 \pm 1.1 \pm 2.9$ & $80.9 \pm 2.2 \pm 3.4$ \\
\hline $0.15<p_{\mathrm{T}}<0.65$ & $85.6 \pm 16.7 \pm 11.0$ & $95.4 \pm 1.8 \pm 3.4$ & $93.9 \pm 1.4 \pm 3.2$ & $87.3 \pm 2.3 \pm 3.1$ & $90.1 \pm 12.6 \pm 4.1$ \\
$0.65<p_{\mathrm{T}}<1.00$ & $97.5 \pm 5.3 \pm 3.8$ & $96.5 \pm 2.2 \pm 3.4$ & $91.8 \pm 1.3 \pm 3.1$ & $89.5 \pm 1.8 \pm 3.1$ & $86.2 \pm 4.2 \pm 3.0$ \\
$1.00<p_{\mathrm{T}}<2.50$ & $98.2 \pm 2.9 \pm 3.3$ & $96.6 \pm 1.8 \pm 3.2$ & $92.5 \pm 1.5 \pm 3.1$ & $90.0 \pm 1.7 \pm 3.1$ & $79.0 \pm 2.8 \pm 2.8$ \\
\hline
\end{tabular}

(b)

\begin{tabular}{|cccccc|}
\hline $\bar{\Lambda} / K_{\mathrm{S}}^{0}$ & $2.0<y<2.5$ & $2.5<y<3.0$ & $3.0<y<3.5$ & $3.5<y<4.0$ & $4.0<y<4.5$ \\
\hline $0.15<p_{\mathrm{T}}<2.50$ & $29.4 \pm 0.6 \pm 2.8$ & $28.4 \pm 0.3 \pm 2.6$ & $28.0 \pm 0.2 \pm 2.5$ & $27.9 \pm 0.3 \pm 2.5$ & $28.7 \pm 0.6 \pm 2.7$ \\
\hline $0.15<p_{\mathrm{T}}<0.65$ & $18.5 \pm 2.7 \pm 2.9$ & $20.0 \pm 0.3 \pm 2.5$ & $19.2 \pm 0.2 \pm 2.3$ & $17.9 \pm 0.4 \pm 2.3$ & $21.1 \pm 1.5 \pm 2.9$ \\
$0.65<p_{\mathrm{T}}<1.00$ & $32.3 \pm 1.3 \pm 2.9$ & $33.3 \pm 0.6 \pm 2.8$ & $32.2 \pm 0.4 \pm 2.7$ & $30.2 \pm 0.5 \pm 2.6$ & $32.2 \pm 1.2 \pm 2.7$ \\
$1.00<p_{\mathrm{T}}<2.50$ & $47.9 \pm 1.1 \pm 3.3$ & $47.5 \pm 0.7 \pm 3.2$ & $45.7 \pm 0.6 \pm 3.2$ & $45.6 \pm 0.7 \pm 3.1$ & $39.5 \pm 1.0 \pm 2.8$ \\
\hline
\end{tabular}

(c)

\begin{tabular}{|ccc|}
\hline $2.0<y<4.5$ & $\bar{\Lambda} / \Lambda$ & $\bar{\Lambda} / K_{\mathrm{S}}^{0}$ \\
\hline $0.15<p_{\mathrm{T}}<0.50$ & $95.0 \pm 1.4 \pm 3.2$ & $16.9 \pm 0.2 \pm 2.3$ \\
$0.50<p_{\mathrm{T}}<0.65$ & $92.9 \pm 1.4 \pm 3.2$ & $23.8 \pm 0.3 \pm 2.4$ \\
$0.65<p_{\mathrm{T}}<0.80$ & $94.0 \pm 1.4 \pm 3.2$ & $29.4 \pm 0.3 \pm 2.5$ \\
$0.80<p_{\mathrm{T}}<1.00$ & $91.9 \pm 1.3 \pm 3.1$ & $35.5 \pm 0.4 \pm 2.7$ \\
$1.00<p_{\mathrm{T}}<1.20$ & $93.1 \pm 1.5 \pm 3.1$ & $41.3 \pm 0.6 \pm 2.9$ \\
$1.20<p_{\mathrm{T}}<2.50$ & $91.8 \pm 1.1 \pm 3.0$ & $48.9 \pm 0.5 \pm 3.2$ \\
\hline
\end{tabular}

Table 6. The production ratios $\bar{\Lambda} / \Lambda$ and $\bar{\Lambda} / K_{\mathrm{S}}^{0}$ without non-prompt corrections at $\sqrt{s}=7 \mathrm{TeV}$ are quoted in percent with statistical and systematic errors as a function of (a) \& (b) rapidity, $y$, and $(\mathrm{c})$ transverse momentum, $p_{\mathrm{T}}[\mathrm{GeV} / c]$. 


\section{The LHCb collaboration}

R. Aaij ${ }^{23}$, B. Adeva ${ }^{36}$, M. Adinolfi ${ }^{42}$, C. Adrover ${ }^{6}$, A. Affolder ${ }^{48}$, Z. Ajaltouni ${ }^{5}$, J. Albrecht ${ }^{37}$, F. Alessio 6,37, M. Alexander ${ }^{47}$, G. Alkhazov ${ }^{29}$, P. Alvarez Cartelle ${ }^{36}$, A.A. Alves Jr ${ }^{22}$, S. Amato ${ }^{2}$, Y. Amhis ${ }^{38}$, J. Anderson ${ }^{39}$, R.B. Appleby ${ }^{50}$, O. Aquines Gutierrez ${ }^{10}$, L. Arrabito ${ }^{53}$, A. Artamonov ${ }^{34}$, M. Artuso ${ }^{52,37}$, E. Aslanides ${ }^{6}$, G. Auriemma ${ }^{22, m}$, S. Bachmann ${ }^{11}$, J.J. Back ${ }^{44}$, D.S. Bailey ${ }^{50}$, V. Balagura ${ }^{30,37}$, W. Baldini ${ }^{16}$, R.J. Barlow ${ }^{50}$, C. Barschel ${ }^{37}$, S. Barsuk $^{7}$, W. Barter ${ }^{43}$, A. Bates ${ }^{47}$, C. Bauer ${ }^{10}$, Th. Bauer ${ }^{23}$, A. Bay ${ }^{38}$, I. Bediaga ${ }^{1}$, K. Belous ${ }^{34}$, I. Belyaev ${ }^{30,37}$, E. Ben-Haim ${ }^{8}$, M. Benayoun ${ }^{8}$, G. Bencivenni ${ }^{18}$, S. Benson ${ }^{46}$, R. Bernet ${ }^{39}$, M.-O. Bettler ${ }^{17,37}$, M. van Beuzekom ${ }^{23}$, A. Bien ${ }^{11}$, S. Bifani ${ }^{12}$, A. Bizzeti ${ }^{17, h}$, P.M. Bjørnstad ${ }^{50}$, T. Blake ${ }^{49}$, F. Blanc ${ }^{38}$, C. Blanks ${ }^{49}$, J. Blouw ${ }^{11}$, S. Blusk ${ }^{52}$, A. Bobrov ${ }^{33}$, V. Bocci ${ }^{22}$, A. Bondar ${ }^{33}$, N. Bondar ${ }^{29}$, W. Bonivento ${ }^{15}$, S. Borghi ${ }^{47}$, A. Borgia ${ }^{52}$, T.J.V. Bowcock ${ }^{48}$, C. Bozzi ${ }^{16}$, T. Brambach ${ }^{9}$, J. van den Brand ${ }^{24}$, J. Bressieux ${ }^{38}$, D. Brett ${ }^{50}$, S. Brisbane ${ }^{51}$, M. Britsch ${ }^{10}$, T. Britton ${ }^{52}$, N.H. Brook ${ }^{42}$, A. Büchler-Germann ${ }^{39}$, I. Burducea ${ }^{28}$, A. Bursche ${ }^{39}$, J. Buytaert ${ }^{37}$, S. Cadeddu ${ }^{15}$, J.M. Caicedo Carvajal ${ }^{37}$, O. Callot ${ }^{7}$, M. Calvi ${ }^{20, j}$, M. Calvo Gomez ${ }^{35, n}$, A. Camboni ${ }^{35}$, P. Campana ${ }^{18,37}$, A. Carbone ${ }^{14}$, G. Carboni ${ }^{21, k}$, R. Cardinale ${ }^{19, i}$, A. Cardini ${ }^{15}$, L. Carson ${ }^{36}$, K. Carvalho Akiba ${ }^{23}$, G. Casse $^{48}$, M. Cattaneo ${ }^{37}$, M. Charles ${ }^{51}$, Ph. Charpentier ${ }^{37}$, N. Chiapolini ${ }^{39}$, X. Cid Vidal ${ }^{36}$, G. Ciezarek ${ }^{49}$, P.E.L. Clarke ${ }^{46,37}$, M. Clemencic ${ }^{37}$, H.V. Cliff ${ }^{43}$, J. Closier ${ }^{37}$, C. Coca ${ }^{28}$, V. Coco ${ }^{23}$, J. Cogan ${ }^{6}$, P. Collins ${ }^{37}$, F. Constantin ${ }^{28}$, G. Conti ${ }^{38}$, A. Contu ${ }^{51}$, M. Coombes ${ }^{42}$, G. Corti ${ }^{37}$, G.A. Cowan ${ }^{38}$, R. Currie ${ }^{46}$, B. D’Almagne ${ }^{7}$, C. D’Ambrosio ${ }^{37}$, P. David ${ }^{8}$, I. De Bonis ${ }^{4}$, S. De Capua ${ }^{21, k}$,

M. De $\operatorname{Cian}^{39}$, F. De Lorenzi ${ }^{12}$, J.M. De Miranda ${ }^{1}$, L. De Paula ${ }^{2}$, P. De Simone ${ }^{18}$, D. Decamp ${ }^{4}$, M. Deckenhoff ${ }^{9}$, H. Degaudenzi ${ }^{38,37}$, M. Deissenroth ${ }^{11}$, L. Del Buono ${ }^{8}$, C. Deplano ${ }^{15}$,

O. Deschamps ${ }^{5}$, F. Dettori ${ }^{15, d}$, J. Dickens ${ }^{43}$, H. Dijkstra ${ }^{37}$, P. Diniz Batista ${ }^{1}$, D. Dossett ${ }^{44}$, A. Dovbnya ${ }^{40}$, F. Dupertuis ${ }^{38}$, R. Dzhelyadin ${ }^{34}$, C. Eames ${ }^{49}$, S. Easo ${ }^{45}$, U. Egede ${ }^{49}$, V. Egorychev ${ }^{30}$, S. Eidelman ${ }^{33}$, D. van Eijk $^{23}$, F. Eisele ${ }^{11}$, S. Eisenhardt ${ }^{46}$, R. Ekelhof ${ }^{9}$, L. Eklund ${ }^{47}$, Ch. Elsasser ${ }^{39}$, D.G. d'Enterria ${ }^{35, o}$, D. Esperante Pereira ${ }^{36}$, L. Estève ${ }^{43}$, A. Falabella ${ }^{16, e}$, E. Fanchini ${ }^{20, j}$, C. Färber ${ }^{11}$, G. Fardell ${ }^{46}$, C. Farinelli ${ }^{23}$, S. Farry ${ }^{12}$, V. Fave ${ }^{38}$, V. Fernandez Albor ${ }^{36}$, M. Ferro-Luzzi ${ }^{37}$, S. Filippov ${ }^{32}$, C. Fitzpatrick ${ }^{46}$, M. Fontana ${ }^{10}$, F. Fontanelli ${ }^{19, i}$, R. Forty ${ }^{37}$, M. Frank ${ }^{37}$, C. Frei ${ }^{37}$, M. Frosini ${ }^{17, f, 37}$, S. Furcas ${ }^{20}$,

A. Gallas Torreira ${ }^{36}$, D. Galli ${ }^{14, c}$, M. Gandelman ${ }^{2}$, P. Gandini ${ }^{51}$, Y. Gao ${ }^{3}$, J-C. Garnier ${ }^{37}$, J. Garofoli ${ }^{52}$, J. Garra Tico ${ }^{43}$, L. Garrido ${ }^{35}$, C. Gaspar ${ }^{37}$, N. Gauvin ${ }^{38}$, M. Gersabeck ${ }^{37}$, T. Gershon ${ }^{44}$, Ph. Ghez ${ }^{4}$, V. Gibson ${ }^{43}$, V.V. Gligorov ${ }^{37}$, C. Göbel $^{54}$, D. Golubkov ${ }^{30}$, A. Golutvin ${ }^{49,30,37}$, A. Gomes ${ }^{2}$, H. Gordon ${ }^{51}$, M. Grabalosa Gándara ${ }^{35}$, R. Graciani Diaz ${ }^{35}$, L.A. Granado Cardoso ${ }^{37}$, E. Graugés ${ }^{35}$, G. Graziani ${ }^{17}$, A. Grecu ${ }^{28}$, S. Gregson ${ }^{43}$, B. Gui ${ }^{52}$, E. Gushchin ${ }^{32}$, Yu. Guz ${ }^{34}$, T. Gys ${ }^{37}$, G. Haefeli ${ }^{38}$, C. Haen ${ }^{37}$, S.C. Haines ${ }^{43}$, T. Hampson ${ }^{42}$, S. Hansmann-Menzemer ${ }^{11}$, R. Harji ${ }^{49}$, N. Harnew ${ }^{51}$, J. Harrison ${ }^{50}$, P.F. Harrison ${ }^{44}$, J. He ${ }^{7}$, V. Heijne ${ }^{23}$, K. Hennessy ${ }^{48}$, P. Henrard ${ }^{5}$, J.A. Hernando Morata ${ }^{36}$, E. van Herwijnen ${ }^{37}$, W. Hofmann ${ }^{10}$, K. Holubyev ${ }^{11}$, P. Hopchev ${ }^{4}$, W. Hulsbergen ${ }^{23}$, P. Hunt ${ }^{51}$, T. Huse ${ }^{48}$, R.S. Huston ${ }^{12}$, D. Hutchcroft ${ }^{48}$, D. Hynds ${ }^{47}$, V. Iakovenko ${ }^{41}$, P. Ilten ${ }^{12}$, J. Imong ${ }^{42}$, R. Jacobsson ${ }^{37}$, A. Jaeger ${ }^{11}$, M. Jahjah Hussein ${ }^{5}$, E. Jans ${ }^{23}$, F. Jansen ${ }^{23}$, P. Jaton ${ }^{38}$, B. Jean-Marie ${ }^{7}$, F. Jing ${ }^{3}$, M. John ${ }^{51}$, D. Johnson ${ }^{51}$, C.R. Jones ${ }^{43}$, B. Jost ${ }^{37}$, S. Kandybei ${ }^{40}$, M. Karacson ${ }^{37}$, T.M. Karbach ${ }^{9}$, J. Keaveney ${ }^{12}$, U. Kerzel ${ }^{37}$, T. Ketel ${ }^{24}$, A. Keune ${ }^{38}$, B. Khanji ${ }^{6}$, Y.M. Kim ${ }^{46}$, M. Knecht ${ }^{38}$, S. Koblitz ${ }^{37}$, P. Koppenburg ${ }^{23}$, A. Kozlinskiy ${ }^{23}$, L. Kravchuk ${ }^{32}$, K. Kreplin ${ }^{11}$, M. Kreps ${ }^{44}$, G. Krocker ${ }^{11}$, P. Krokovny ${ }^{11}$, F. Kruse ${ }^{9}$, K. Kruzelecki ${ }^{37}$, M. Kucharczyk ${ }^{20,25}$, S. Kukulak ${ }^{25}$, R. Kumar ${ }^{14,37}$, T. Kvaratskheliya ${ }^{30,37}$, V.N. La Thi ${ }^{38}$, D. Lacarrere ${ }^{37}$, G. Lafferty ${ }^{50}$, A. Lai ${ }^{15}$, D. Lambert ${ }^{46}$, R.W. Lambert ${ }^{37}$, E. Lanciotti ${ }^{37}$, G. Lanfranchi ${ }^{18}$, C. Langenbruch ${ }^{11}$, T. Latham ${ }^{44}$, R. Le Gac ${ }^{6}$, J. van Leerdam ${ }^{23}$, J.-P. Lees ${ }^{4}$, 


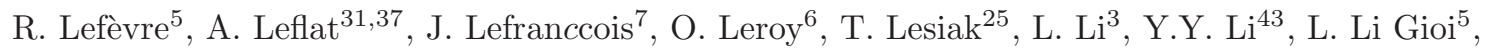
M. Lieng ${ }^{9}$, R. Lindner ${ }^{37}$, C. Linn ${ }^{11}$, B. Liu ${ }^{3}$, G. Liu ${ }^{37}$, J.H. Lopes ${ }^{2}$, E. Lopez Asamar ${ }^{35}$,

N. Lopez-March ${ }^{38}$, J. Luisier ${ }^{38}$, F. Machefert ${ }^{7}$, I.V. Machikhiliyan ${ }^{4,30}$, F. Maciuc ${ }^{10}$, O. Maev ${ }^{29,37}$, J. Magnin ${ }^{1}$, S. Malde ${ }^{51}$, R.M.D. Mamunur ${ }^{37}$, G. Manca ${ }^{15, d}$, G. Mancinelli ${ }^{6}$, N. Mangiafave ${ }^{43}$, U. Marconi ${ }^{14}$, R. Märki ${ }^{38}$, J. Marks ${ }^{11}$, G. Martellotti ${ }^{22}$, A. Martens ${ }^{7}$, L. Martin ${ }^{51}$,

A. Martín Sánchez ${ }^{7}$, D. Martinez Santos ${ }^{37}$, A. Massafferri ${ }^{1}$, Z. Mathe ${ }^{12}$, C. Matteuzzi ${ }^{20}$, M. Matveev ${ }^{29}$, E. Maurice 6 , B. Maynard ${ }^{52}$, A. Mazurov ${ }^{32,16,37}$, G. McGregor ${ }^{50}$, R. McNulty ${ }^{12}$, C. Mclean ${ }^{14}$, M. Meissner ${ }^{11}$, M. Merk ${ }^{23}$, J. Merkel ${ }^{9}$, R. Messi ${ }^{21, k}$, S. Miglioranzi ${ }^{37}$,

D.A. Milanes ${ }^{13,37}$, M.-N. Minard ${ }^{4}$, S. Monteil ${ }^{5}$, D. Moran ${ }^{12}$, P. Morawski ${ }^{25}$, J.V. Morris ${ }^{45}$,

R. Mountain ${ }^{52}$, I. Mous ${ }^{23}$, F. Muheim ${ }^{46}$, K. Müller ${ }^{39}$, R. Muresan ${ }^{28,38}$, B. Muryn ${ }^{26}$, M. Musy $^{35}$,

P. Naik ${ }^{42}$, T. Nakada ${ }^{38}$, R. Nandakumar ${ }^{45}$, J. Nardulli ${ }^{45}$, I. Nasteva ${ }^{1}$, M. Nedos ${ }^{9}$, M. Needham ${ }^{46}$,

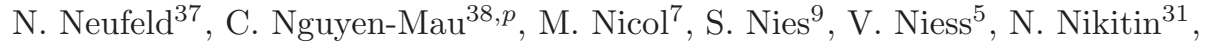

A. Oblakowska-Mucha ${ }^{26}$, V. Obraztsov ${ }^{34}$, S. Oggero ${ }^{23}$, S. Ogilvy ${ }^{47}$, O. Okhrimenko ${ }^{41}$,

R. Oldeman ${ }^{15, d}$, M. Orlandea ${ }^{28}$, J.M. Otalora Goicochea ${ }^{2}$, P. Owen ${ }^{49}$, B. Pal ${ }^{52}$, J. Palacios ${ }^{39}$,

M. Palutan ${ }^{18}$, J. Panman ${ }^{37}$, A. Papanestis ${ }^{45}$, M. Pappagallo ${ }^{13, b}$, C. Parkes ${ }^{47,37}$, C.J. Parkinson ${ }^{49}$,

G. Passaleva ${ }^{17}$, G.D. Patel ${ }^{48}$, M. Patel ${ }^{49}$, S.K. Paterson ${ }^{49}$, G.N. Patrick ${ }^{45}$, C. Patrignani ${ }^{19, i}$,

C. Pavel-Nicorescu ${ }^{28}$, A. Pazos Alvarez ${ }^{36}$, A. Pellegrino ${ }^{23}$, G. Penso $^{22, l}$, M. Pepe Altarelli ${ }^{37}$,

S. Perazzini ${ }^{14, c}$, D.L. Perego ${ }^{20, j}$, E. Perez Trigo ${ }^{36}$, A. Pérez-Calero Yzquierdo ${ }^{35}$, P. Perret ${ }^{5}$,

M. Perrin-Terrin ${ }^{6}$, G. Pessina ${ }^{20}$, A. Petrella ${ }^{16,37}$, A. Petrolini ${ }^{19, i}$, B. Pie Valls ${ }^{35}$, B. Pietrzyk ${ }^{4}$,

T. Pilar ${ }^{44}$, D. Pinci ${ }^{22}$, R. Plackett ${ }^{47}$, S. Playfer ${ }^{46}$, M. Plo Casasus ${ }^{36}$, G. Polok ${ }^{25}$,

A. Poluektov ${ }^{44,33}$, E. Polycarpo ${ }^{2}$, D. Popov ${ }^{10}$, B. Popovici ${ }^{28}$, C. Potterat ${ }^{35}$, A. Powell ${ }^{51}$,

T. du Pree ${ }^{23}$, J. Prisciandaro ${ }^{38}$, V. Pugatch ${ }^{41}$, A. Puig Navarro ${ }^{35}$, W. Qian ${ }^{52}$, J.H. Rademacker ${ }^{42}$,

B. Rakotomiaramanana ${ }^{38}$, I. Raniuk ${ }^{40}$, G. Raven ${ }^{24}$, S. Redford ${ }^{51}$, M.M. Reid ${ }^{44}$, A.C. dos Reis ${ }^{1}$,

S. Ricciardi ${ }^{45}$, K. Rinnert ${ }^{48}$, D.A. Roa Romero ${ }^{5}$, P. Robbe ${ }^{7}$, E. Rodrigues ${ }^{47}$, F. Rodrigues ${ }^{2}$,

P. Rodriguez Perez ${ }^{36}$, G.J. Rogers ${ }^{43}$, V. Romanovsky ${ }^{34}$, J. Rouvinet ${ }^{38}$, T. Ruf ${ }^{37}$, H. Ruiz ${ }^{35}$,

G. Sabatino ${ }^{21, k}$, J.J. Saborido Silva ${ }^{36}$, N. Sagidova ${ }^{29}$, P. Sail ${ }^{47}$, B. Saitta ${ }^{15, d}$, C. Salzmann ${ }^{39}$,

M. Sannino ${ }^{19, i}$, R. Santacesaria ${ }^{22}$, R. Santinelli ${ }^{37}$, E. Santovetti ${ }^{21, k}$, M. Sapunov ${ }^{6}$, A. Sarti ${ }^{18, l}$,

C. Satriano ${ }^{22, m}$, A. Satta ${ }^{21}$, M. Savrie ${ }^{16, e}$, D. Savrina ${ }^{30}$, P. Schaack ${ }^{49}$, M. Schiller ${ }^{11}$, S. Schleich ${ }^{9}$,

M. Schmelling ${ }^{10}$, B. Schmidt ${ }^{37}$, O. Schneider ${ }^{38}$, A. Schopper ${ }^{37}$, M.-H. Schune ${ }^{7}$, R. Schwemmer ${ }^{37}$,

A. Sciubba ${ }^{18, l}$, M. Seco ${ }^{36}$, A. Semennikov ${ }^{30}$, K. Senderowska ${ }^{26}$, I. Sepp ${ }^{49}$, N. Serra ${ }^{39}$, J. Serrano ${ }^{6}$,

P. Seyfert ${ }^{11}$, B. Shao ${ }^{3}$, M. Shapkin ${ }^{34}$, I. Shapoval ${ }^{40,37}$, P. Shatalov ${ }^{30}$, Y. Shcheglov ${ }^{29}$, T. Shears ${ }^{48}$,

L. Shekhtman ${ }^{33}$, O. Shevchenko ${ }^{40}$, V. Shevchenko ${ }^{30}$, A. Shires ${ }^{49}$, R. Silva Coutinho ${ }^{54}$,

H.P. Skottowe ${ }^{43}$, T. Skwarnicki ${ }^{52}$, A.C. Smith $^{37}$, N.A. Smith ${ }^{48}$, K. Sobczak ${ }^{5}$, F.J.P. Soler ${ }^{47}$,

A. Solomin ${ }^{42}$, F. Soomro ${ }^{49}$, B. Souza De Paula ${ }^{2}$, B. Spaan ${ }^{9}$, A. Sparkes ${ }^{46}$, P. Spradlin ${ }^{47}$,

F. Stagni ${ }^{37}$, S. Stahl ${ }^{11}$, O. Steinkamp ${ }^{39}$, S. Stoica ${ }^{28}$, S. Stone ${ }^{52,37}$, B. Storaci ${ }^{23}$, M. Straticiuc ${ }^{28}$,

U. Straumann ${ }^{39}$, N. Styles ${ }^{46}$, S. Swientek ${ }^{9}$, M. Szczekowski ${ }^{27}$, P. Szczypka ${ }^{38}$, T. Szumlak ${ }^{26}$,

S. T'Jampens ${ }^{4}$, E. Teodorescu ${ }^{28}$, F. Teubert ${ }^{37}$, C. Thomas ${ }^{51,45}$, E. Thomas ${ }^{37}$, J. van Tilburg ${ }^{11}$,

V. Tisserand ${ }^{4}$, M. Tobin ${ }^{39}$, S. Topp-Joergensen ${ }^{51}$, M.T. Tran $^{38}$, A. Tsaregorodtsev ${ }^{6}$, N. Tuning ${ }^{23}$,

A. Ukleja ${ }^{27}$, P. Urquijo ${ }^{52}$, U. Uwer ${ }^{11}$, V. Vagnoni ${ }^{14}$, G. Valenti ${ }^{14}$, R. Vazquez Gomez ${ }^{35}$,

P. Vazquez Regueiro ${ }^{36}$, S. Vecchi ${ }^{16}$, J.J. Velthuis ${ }^{42}$, M. Veltri ${ }^{17, g}$, K. Vervink ${ }^{37}$, B. Viaud ${ }^{7}$,

I. Videau ${ }^{7}$, X. Vilasis-Cardona ${ }^{35, n}$, J. Visniakov ${ }^{36}$, A. Vollhardt ${ }^{39}$, D. Voong ${ }^{42}$, A. Vorobyev ${ }^{29}$,

H. $\operatorname{Voss}^{10}$, K. Wacker ${ }^{9}$, S. Wandernoth ${ }^{11}$, J. Wang ${ }^{52}$, D.R. Ward ${ }^{43}$, A.D. Webber ${ }^{50}$,

D. Websdale ${ }^{49}$, M. Whitehead ${ }^{44}$, D. Wiedner ${ }^{11}$, L. Wiggers ${ }^{23}$, G. Wilkinson ${ }^{51}$, M.P. Williams ${ }^{44,45}$,

M. Williams ${ }^{49}$, F.F. Wilson ${ }^{45}$, J. Wishahi ${ }^{9}$, M. Witek ${ }^{25}$, W. Witzeling ${ }^{37}$, S.A. Wotton ${ }^{43}$,

K. Wyllie ${ }^{37}$, Y. Xie ${ }^{46}$, F. Xing ${ }^{51}$, Z. Yang ${ }^{3}$, R. Young ${ }^{46}$, O. Yushchenko ${ }^{34}$, M. Zavertyaev ${ }^{10, a}$,

L. Zhang ${ }^{52}$, W.C. Zhang ${ }^{12}$, Y. Zhang ${ }^{3}$, A. Zhelezov ${ }^{11}$, L. Zhong ${ }^{3}$, E. Zverev ${ }^{31}$, A. Zvyagin ${ }^{37}$. 
${ }^{1}$ Centro Brasileiro de Pesquisas Físicas (CBPF), Rio de Janeiro, Brazil

${ }^{2}$ Universidade Federal do Rio de Janeiro (UFRJ), Rio de Janeiro, Brazil

${ }^{3}$ Center for High Energy Physics, Tsinghua University, Beijing, China

${ }^{4}$ LAPP, Université de Savoie, CNRS/IN2P3, Annecy-Le-Vieux, France

${ }^{5}$ Clermont Université, Université Blaise Pascal, CNRS/IN2P3, LPC, Clermont-Ferrand, France

${ }^{6}$ CPPM, Aix-Marseille Université, CNRS/IN2P3, Marseille, France

${ }^{7}$ LAL, Université Paris-Sud, CNRS/IN2P3, Orsay, France

${ }^{8}$ LPNHE, Université Pierre et Marie Curie, Université Paris Diderot, CNRS/IN2P3, Paris,

France

${ }^{9}$ Fakultät Physik, Technische Universität Dortmund, Dortmund, Germany

${ }^{10}$ Max-Planck-Institut für Kernphysik (MPIK), Heidelberg, Germany

${ }^{11}$ Physikalisches Institut, Ruprecht-Karls-Universität Heidelberg, Heidelberg, Germany

${ }^{12}$ School of Physics, University College Dublin, Dublin, Ireland

${ }^{13}$ Sezione INFN di Bari, Bari, Italy

${ }^{14}$ Sezione INFN di Bologna, Bologna, Italy

${ }^{15}$ Sezione INFN di Cagliari, Cagliari, Italy

${ }^{16}$ Sezione INFN di Ferrara, Ferrara, Italy

${ }^{17}$ Sezione INFN di Firenze, Firenze, Italy

${ }^{18}$ Laboratori Nazionali dell'INFN di Frascati, Frascati, Italy

${ }^{19}$ Sezione INFN di Genova, Genova, Italy

${ }^{20}$ Sezione INFN di Milano Bicocca, Milano, Italy

${ }^{21}$ Sezione INFN di Roma Tor Vergata, Roma, Italy

${ }^{22}$ Sezione INFN di Roma La Sapienza, Roma, Italy

${ }^{23}$ Nikhef National Institute for Subatomic Physics, Amsterdam, Netherlands

${ }^{24}$ Nikhef National Institute for Subatomic Physics and Vrije Universiteit, Amsterdam, Netherlands

${ }^{25}$ Henryk Niewodniczanski Institute of Nuclear Physics Polish Academy of Sciences, Cracow,

Poland

${ }^{26}$ Faculty of Physics 83 Applied Computer Science, Cracow, Poland

${ }^{27}$ Soltan Institute for Nuclear Studies, Warsaw, Poland

${ }^{28}$ Horia Hulubei National Institute of Physics and Nuclear Engineering, Bucharest-Magurele, Romania

${ }^{29}$ Petersburg Nuclear Physics Institute (PNPI), Gatchina, Russia

${ }^{30}$ Institute of Theoretical and Experimental Physics (ITEP), Moscow, Russia

${ }^{31}$ Institute of Nuclear Physics, Moscow State University (SINP MSU), Moscow, Russia

${ }^{32}$ Institute for Nuclear Research of the Russian Academy of Sciences (INR RAN), Moscow, Russia

${ }^{33}$ Budker Institute of Nuclear Physics (SB RAS) and Novosibirsk State University, Novosibirsk, Russia

${ }^{34}$ Institute for High Energy Physics (IHEP), Protvino, Russia

${ }^{35}$ Universitat de Barcelona, Barcelona, Spain

${ }^{36}$ Universidad de Santiago de Compostela, Santiago de Compostela, Spain

${ }^{37}$ European Organization for Nuclear Research (CERN), Geneva, Switzerland

${ }^{38}$ Ecole Polytechnique Fédérale de Lausanne (EPFL), Lausanne, Switzerland

${ }^{39}$ Physik-Institut, Universität Zürich, Zürich, Switzerland

${ }^{40}$ NSC Kharkiv Institute of Physics and Technology (NSC KIPT), Kharkiv, Ukraine

${ }^{41}$ Institute for Nuclear Research of the National Academy of Sciences (KINR), Kyiv, Ukraine

${ }^{42}$ H.H. Wills Physics Laboratory, University of Bristol, Bristol, United Kingdom

${ }^{43}$ Cavendish Laboratory, University of Cambridge, Cambridge, United Kingdom

${ }^{44}$ Department of Physics, University of Warwick, Coventry, United Kingdom 
${ }^{45}$ STFC Rutherford Appleton Laboratory, Didcot, United Kingdom

${ }^{46}$ School of Physics and Astronomy, University of Edinburgh, Edinburgh, United Kingdom

${ }^{47}$ School of Physics and Astronomy, University of Glasgow, Glasgow, United Kingdom

${ }^{48}$ Oliver Lodge Laboratory, University of Liverpool, Liverpool, United Kingdom

${ }^{49}$ Imperial College London, London, United Kingdom

${ }^{50}$ School of Physics and Astronomy, University of Manchester, Manchester, United Kingdom

${ }^{51}$ Department of Physics, University of Oxford, Oxford, United Kingdom

${ }^{52}$ Syracuse University, Syracuse, NY, United States

${ }^{53}$ CC-IN2P3, CNRS/IN2P3, Lyon-Villeurbanne, France, associated member

${ }^{54}$ Pontifícia Universidade Católica do Rio de Janeiro (PUC-Rio), Rio de Janeiro, Brazil, associated to ${ }^{2}$

${ }^{a}$ P.N. Lebedev Physical Institute, Russian Academy of Science (LPI RAS), Moscow, Russia

${ }^{b}$ Università di Bari, Bari, Italy

${ }^{c}$ Università di Bologna, Bologna, Italy

${ }^{d}$ Università di Cagliari, Cagliari, Italy

${ }^{e}$ Università di Ferrara, Ferrara, Italy

${ }^{f}$ Università di Firenze, Firenze, Italy

${ }^{g}$ Università di Urbino, Urbino, Italy

${ }^{h}$ Università di Modena e Reggio Emilia, Modena, Italy

${ }^{i}$ Università di Genova, Genova, Italy

${ }^{j}$ Università di Milano Bicocca, Milano, Italy

${ }^{k}$ Università di Roma Tor Vergata, Roma, Italy

${ }^{l}$ Università di Roma La Sapienza, Roma, Italy

${ }^{m}$ Università della Basilicata, Potenza, Italy

${ }^{n}$ LIFAELS, La Salle, Universitat Ramon Llull, Barcelona, Spain

${ }^{\circ}$ Institució Catalana de Recerca i Estudis Avanccats (ICREA), Barcelona, Spain

${ }^{p}$ Hanoi University of Science, Hanoi, Viet Nam

Open Access. This article is distributed under the terms of the Creative Commons Attribution Noncommercial License which permits any noncommercial use, distribution, and reproduction in any medium, provided the original author(s) and source are credited.

\section{References}

[1] STAR collaboration, B.I. Abelev et al., Strange particle production in $p+p$ collisions at $\sqrt{s}=200 \mathrm{GeV}$, Phys. Rev. C 75 (2007) 064901 [nucl-ex/0607033] [SPIRES].

[2] K. Aamodt et al., Strange particle production in proton-proton collisions at $\sqrt{s}=0.9 \mathrm{TeV}$ with ALICE at the LHC, Eur. Phys. J. C 71 (2011) 1594 [arXiv:1012.3257] [SPIRES].

[3] CMS collaboration, V. Khachatryan et al., Strange particle production in pp collisions at $\sqrt{s}=0.9$ and $7 \mathrm{TeV}$, JHEP 05 (2011) 064 [arXiv: 1102.4282] [SPIRES].

[4] LHCb collaboration, R. Aaij et al., Prompt $K_{S}^{0}$ production in pp collisions at $\sqrt{s}=0.9 \mathrm{TeV}$, Phys. Lett. B 693 (2010) 69 [arXiv:1008.3105] [SPIRES].

[5] LHCb collaboration, A.A. Alves et al., The LHCb Detector at the $\mathrm{LHC}$, 2008 JINST 3 S08005 [SPIRES]. 
[6] I. Belyaev et al., Handling of the generation of primary events in Gauss, the LHCb simulation framework, IEEE Nucl. Sci. Symp. Conf. Rec. (2010) 1155.

[7] T. Sjöstrand, S. Mrenna and P.Z. Skands, PYTHIA 6.4 Physics and Manual, JHEP 05 (2006) 026 [hep-ph/0603175] [SPIRES].

[8] D.J. Lange, The EvtGen particle decay simulation package, Nucl. Instrum. Meth. A 462 (2001) 152 [SPIRES].

[9] P. Golonka and Z. Was, PHOTOS Monte Carlo: A Precision tool for QED corrections in Z and $W$ decays, Eur. Phys. J. C 45 (2006) 97 [hep-ph/0506026] [SPIRES].

[10] GEANT4 collaboration, S. Agostinelli et al., GEANT4: A simulation toolkit, Nucl. Instrum. Meth. A 506 (2003) 250 [SPIRES].

[11] P.Z. Skands, Tuning Monte Carlo Generators: The Perugia Tunes, Phys. Rev. D 82 (2010) 074018 [arXiv: 1005.3457] [SPIRES].

[12] T. Sjöstrand, S. Mrenna and P.Z. Skands, A Brief Introduction to PYTHIA 8.1, Comput. Phys. Commun. 178 (2008) 852 [arXiv: 0710.3820] [SPIRES].

[13] S. Navin, Diffraction in PYTHIA, arXiv:1005.3894 [SPIRES].

[14] Particle Data Group collaboration, K. Nakamura et al., Review of particle physics, J. Phys. G 37 (2010) 075021 [SPIRES]. 